\title{
Down-Regulation of Mitochondrial Metabolism after Tendon Release Primes Lipid Accumulation in Rotator Cuff Muscle
}

\author{
Martin Flück, ${ }^{*}$ Daniel Fitze, ${ }^{*}$ Severin Ruoss, ${ }^{*}$ Paola Valdivieso, ${ }^{*}$ Brigitte von Rechenberg, ${ }^{\dagger}$ Anna Bratus-Neuenschwander, \\ Lennart Opitz, ${ }^{\ddagger}$ Junmin Hu, ${ }^{\ddagger}$ Endre Laczko, ${ }^{\ddagger}$ Karl Wieser, ${ }^{\S}$ and Christian Gerber
}

From the Laboratory for Muscle Plasticity, ${ }^{*}$ Department of Orthopedics, ${ }^{\S}$ Balgrist University Hospital, the Vetsuisse Faculty, ${ }^{\dagger}$ Musculoskeletal Research Unit, Competence Center for Applied Biotechnology, and the Functional Genomics Center Zurich, ${ }^{\ddagger}$ ETH Zurich, University of Zurich, Zurich, Switzerland

\author{
Accepted for publication \\ March 26, 2020. \\ Address correspondence to \\ Martin Flück, Ph.D., Depart- \\ ment of Orthopedics, University \\ of Zurich, Balgrist Campus, \\ Lengghalde 5, Zurich 8008, \\ Switzerland. E-mail: martin. \\ flueck@balgrist.ch.
}

\begin{abstract}
Atrophy and fat accumulation are debilitating aspects of muscle diseases and are rarely prevented. Using a vertical approach combining anatomic techniques with omics methodology in a tenotomyinduced sheep model of rotator cuff disease, we tested whether mitochondrial dysfunction is implicated in muscle wasting and perturbed lipid metabolism, speculating that both can be prevented by the stimulation of $\beta$-oxidation with L-carnitine. The infraspinatus muscle lost $22 \%$ of its volume over the first 6 weeks after tenotomy before the area-percentage of lipid increased from $8 \%$ to $18 \%$ at week 16 . Atrophy was associated with the down-regulation of mitochondrial transcripts and protein and a slowto-fast shift in muscle composition. Correspondingly, amino acid levels were increased 2 weeks after tendon release, when the levels of high-energy phosphates and glycerophospholipids were lowered. LCarnitine administration $(0.9 \mathrm{~g} / \mathrm{kg}$ per day) prevented atrophy over the first 2 weeks, and mitigated alterations of glutamate, glycerophospholipids, and carnitine levels in released muscle, but did not prevent the level decrease in high-energy phosphates or protein constituents of mitochondrial respiration, promoting the accumulation of longer lipids with an increasing saturation. We conclude that the early phase of infraspinatus muscle degeneration after tendon release involves the elimination of oxidative characteristics associated with an aberrant accumulation of lipid species but is largely unrelated to the prevention of atrophy with oral L-carnitine administration. (Am J Pathol 2020, 190: 1513 -1529; https://doi.org/10.1016/j.ajpath.2020.03.019)
\end{abstract}

Rotator cuff disease is a frequent problem, arising subsequent to the distal rupture of a rotator cuff tendon. ${ }^{1}$ In consequence, the affected shoulder has a compromised ability to abduct, lift, or rotate the arm. ${ }^{2}$ In order to restore shoulder functionality, the ruptured tendon may be reattached to the humerus via an anchor during arthroscopic surgery. ${ }^{3,4}$ However, the restoration of rotator cuff function by the reattachment of a detached tendon may be incomplete, or impossible, because the implicated muscle is subjected to volume loss and a subsequently increased fraction of fat (fatty atrophy). ${ }^{5-7}$ The subsequently reduced length and increased muscle stiffness interfere with proper attachment and healing of the reattached muscle-tendon construct. $^{8}$ Based on clinical observations, the window of opportunity for successful rotator cuff repair is estimated to situate in the first 3 to 6 weeks, ${ }^{9-11}$ possibly reflecting the course of muscle deterioration. For instance, experimental studies support the view that rotator cuff muscle deterioration after tendon rupture occurs in a first phase of partially reversible disuse-related muscle atrophy, which is followed by a degenerative phase comprising changes in muscle fiber architecture and the proliferation of connective tissue and fat accumulation. $^{12,13}$

\footnotetext{
Supported by Swiss National Science Foundation (Switzerland) grant 149786 (M.F.) and a Resortho Foundation (Switzerland) grant (M.F.).

Disclosures: None declared.

Current address of D.F., On Your Marks AG, Cham, Switzerland; of S.R., Department of Orthopedic Surgery, University of California, La Jolla, San Diego, California.
} 
The cellular mechanisms leading to skeletal muscle degeneration with tendon rupture involve the removal of myofibrillar material and an increased content of connective tissue and embedded fat cells. ${ }^{14-16}$ The implicated cellular events have been described in terms of the up-regulation of proteolytic and adipogenic factors, the latter involving increases in adipocytes through the transdifferentiation of inherent fibroblast-associated precursors and infiltrating macrophages. ${ }^{15,17}$ However, this description leaves a critical gap regarding the underlying metabolic processes that connect the anabolic changes in rotator cuff muscle after tendon rupture with the accumulation of lipid that manifests at the cellular and radiologic levels. ${ }^{18}$ A linear relationship between fat fraction and the reduced area-percentage of fast-type muscle fibers in sheep infraspinatus muscle 16 weeks after tendon release ${ }^{13}$ indicates that fiber-related metabolic substrates are quantitatively related to enhanced lipid synthesis in atrophying rotator cuff muscle. Unaddressed mechanistic points especially refer to the questions of whether, and how, amino acids being liberated with the degeneration of muscle fibers in a torn rotator cuff, may serve as a substrate for lipid synthesis. ${ }^{19}$

A reduced capacity for mitochondrial metabolism has been shown to play an important part in atrophy and lipid accumulation in other models of muscle degeneration. For instance, the degradation of mitochondria and associated alterations in signaling are part of the muscle atrophy process, ${ }^{20,21}$ and especially a diminished mitochondrial capacity has been tied to the accumulation of lipid in skeletal muscle (reviewed by Hoeks and Schrauwen ${ }^{22}$ ). A down-regulated expression of genes that define the metabolic (ie, mitochondrial) and slow contractile muscle phenotype has been associated with disuse atrophy in rat and human muscle..$^{20,23-26}$ The involvement of mitochondria in muscle atrophy is highlighted by the mitigation of reductions in the muscle weight, muscle fiber cross-sectional area, and expression of genes for mitochondrial oxidative metabolism and protein turnover, in rat models of muscle disuse by supplementation with activators of mitochondrial metabolism, L-carnitine and its derivative acetyl-L-carnitine. ${ }^{27-31}$ Whether lipid accumulation and muscle protein degradation with rotator cuff disease effectively involve mitochondrial alterations, or dysfunction, as suggested during aging, ${ }^{32}$ and whether this is associated with inactivity, ${ }^{33}$ are not understood.

We reasoned that the underrated apprehension of the role of mitochondrial metabolism in fatty atrophy reflects the fact that molecular aspects of rotator cuff disease are typically assessed with a narrow focus on late time points of the pathology, and in rodent models, which reflect the active course and degree of rotator cuff degeneration in humans only to a limited degree. ${ }^{13,34,35}$ For instance, the involvement of mitochondria in rotator cuff disease had, until recently, ${ }^{36}$ been investigated in only a single study in a model that reproduced the clinically observed degree of fat accumulation, and at a time point reflective of a chronically torn rotator cuff, ${ }^{37}$ identifying that mitochondrial volume density per muscle fiber in sheep infraspinatus muscle is not affected when a massive accumulation of fat is established.

The purpose of this study was therefore to investigate the early metabolic processes that precede, and lead to, fatty atrophy of rotator cuff muscle after experimental release of its tendon in sheep, ${ }^{13,37}$ with an explorative multilevel approach. In a first hypothesis-generating part, taking the possible main role of mitochondrial processes into consideration, we addressed the extent to which global alterations of mitochondria-related transcript expression, and metabolite and lipid levels, take place in rotator cuff muscle within the early phase of response to tendon release, before irreversible alterations manifest at the macroscopic level. Subsequently the extent to which nutraceutical treatment with L-carnitine would halt early signs of muscle degeneration was assessed.

\section{Materials and Methods}

\section{Design}

Animal experiments were performed according to Swiss law on animal welfare (TSch455) and with approval of the Veterinary Office of the Canton (Zurich, Switzerland; protocols ZH219/2014 and ZH72/2013).

Female Swiss Alpine sheep (Ovis aries) were subjected to release of the infraspinatus tendon for 2, 6 , and 16 weeks, for: (1) characterization of the course of infraspinatus muscle degeneration based on measurements of molecular parameters (emphasis on mitochondrial metabolism) and cellular parameters on collected bioptic material and radiologic imaging; and (2) study of the effects of the oral administration of L-carnitine (Supplemental Figure S1). Six sheep per group were studied at 2 weeks (T2 group; means \pm SEM age and weight, $16.6 \pm 0.0$ months and $59.7 \pm 2.5 \mathrm{~kg}$ ), 2 to 6 weeks (T2-6 group; means \pm SEM age and weight, $26.7 \pm 0.6$ months and $63.3 \pm 1.4 \mathrm{~kg}$ ), and 16 weeks (T16; means \pm SEM age and weight, $23.2 \pm 1.2$ months and $45.3 \pm 4.8 \mathrm{~kg}$ ) after tendon release. L-Carnitine was administered starting 1 week prior to tendon release in another group of sheep (T2-C group; $29.6 \pm 0.3$ months and $59.9 \pm 2.4 \mathrm{~kg} ; n=3$ ). Aspects of the anatomic data (ie, the muscle volume and histologic examination) that serve as the biological background for this study have been reported previously in other form for the T2-6 and T16 groups. ${ }^{13,38}$

Computed tomography and/or magnetic resonance imaging were performed immediately after tendon release and at the end of the experiment, in the upper body of anesthetized sheep, to estimate the volume and compositional alterations in the released infraspinatus muscle and its contralateral control. Additionally, in group T16, magnetic resonance imaging was performed at 6 weeks after tendon release. Biopsy samples (approximately $50 \mathrm{mg}$ ) were collected intraoperatively with 5-mm Bergstrom needles (Dixon instruments, Wickford, UK) from the lateral aspect within the belly of the operated infraspinatus muscle immediately before tendon release (pre), and from the 
released muscle and its contralateral control at the end of the experiment; samples were frozen in melting isopentane. Sites being selected for repeated biopsy sampling were separated by at least $2 \mathrm{~cm}$ from the previous collection site. This distance was ensured by the insertion of a suture in the fascia above the site selected for the previous biopsy sampling. The sampled tissue was stored at $-80^{\circ} \mathrm{C}$ until subjected to histologic, transcriptomic, and protein analysis. Additional tissue pieces were extracted with a scalpel (approximately $500 \mathrm{mg}$ ) from the lateral aspect within the belly of the released and contralateral infraspinatus muscle that were excised and frozen at the end of the experiment. These samples were used for the metabolomic/lipidomic measurements.

\section{Tendon Release and Animal Care}

The right shoulder of each sheep was subjected to release of the infraspinatus tendon by osteotomy of the greater tuberosity, as described. ${ }^{13}$ Animals were housed in standard housing, monitored for health, and medicated to control eventual pain and discomfort. L-Carnitine (Carnipure tartrate; Lonza, Basel, Switzerland) was dissolved in tap water and administered with the help of a syringe at a daily dose of $0.9 \mathrm{~g} / \mathrm{kg}$. The dose applied corresponded to the dose of the related compound, acetyl-L-carnitine, effective in reducing the degeneration of slow oxidative muscle fibers with 2 weeks of disuse. ${ }^{27}$ During the days after tendon release, animals were confined to a quiet area with limited space for gait activities. At the end of the experiment, euthanasia was performed.

\section{Transcriptomics}

Gene expression was analyzed by RNA sequencing and expressed as a concentration per biopsy volume essentially as described. ${ }^{13}$ In brief, total RNA was isolated from $15 \mathrm{~mm}^{3}$ of cryosections for three biological replicas for the pre and post time points from the T2 and T16 groups using the RNeasy Kit (Qiagen, Basel, Switzerland) and verified for quantity and quality using the Qubit 1.0 RNA BR Assay Kit (Thermo Fisher Scientific, Waltham, MA) and Agilent RNA 6000 Nano Kit with the Agilent 2100 Bioanalyzer (Agilent Technologies, Santa Clara, CA), respectively. RNA libraries were prepared from $1 \mu \mathrm{g}$ of total RNA using the Illumina TruSeq Stranded total RNA Sample Preparation Kit (Illumina, San Diego, CA); quantity and quality of the libraries were checked using quantitative PCR (Roche, Basel, Switzerland) with Illumina adapter-specific primers and Agilent 2200 TapeStation, respectively. Diluted indexed libraries $(10 \mathrm{nmol} / \mathrm{L})$ were pooled and used for cluster generation according to the manufacturer's recommendations using the Illumina TruSeq SR Cluster Kit v3-cBot-HS reagents and sequenced on Illumina HiSeq 2500 [single-read approach $(1 \times 100 \mathrm{bp})$ with 20 to 30 million reads per sample]. Raw reads were cleaned by removing adapter sequences and the first three and last four bases. The resulting reads were aligned, that is, annotated, respective to the current build of the sheep genome (Oar version 3.1.75; Ensembl, https://useast.ensembl.org, last accessed September 9, 2019) using STAR aligner software version 2.3.0e. ${ }^{39}$ RNA signals were normalized to the total RNA signal intensity per analyzed sample, and multiplied with the amount of RNA being analyzed per muscle biopsy (ie, concentration), to reveal relative concentrations per species. Data sets were deposited in the Gene Expression Omnibus database (http://www.ncbi.nlm.nih. gov/geo; accession number GSE144394).

\section{Protein Analysis}

The abundance of selected proteins for gene ontologies, the transcript levels of which were affected by 2 weeks after tendon release, was analyzed by separation of $10 \mu \mathrm{g}$ of total muscle protein in homogenate by $12 \%$ SDS-PAGE, immunoblot analysis with validated antibodies, and enhanced chemiluminescence-based detection, as described. ${ }^{13,38}$ Specifically, this process concerned the detection of protein constituents of mitochondrial respiratory chain complexes I to $\mathrm{V}$ [I, NADH:ubiquinone oxidoreductase subunit A9 (NDUFA9); II, succinate dehydrogenase complex flavoprotein subunit A (SDHA); III, ubiquinol-cytochrome c reductase core protein 1 (UQCRC1); IV, cytochrome c oxidase subunit 4I1 (COX4I1); $\mathrm{V}$, ATP synthase F1 subunit $\alpha$ (ATP5A1)] through the use of the anti-OxPhos Complex Kit (InvitroGen, Carlsbad, CA), or detection of slow-type myosin heavy chain, fast-type myosin heavy chain with the primary antibodies [catalog number MAB1628 (Merck \& Cie, Altdorf, Switzerland); My-32 (Sigma-Aldrich, Buchs, Switzerland)], followed by incubation with horseradish peroxidase-coupled secondary antimouse IgG (Fab Specific-Peroxidase; Sigma-Aldrich, St. Louis, MO). Equal protein loading and blotting were verified based on Ponceau S staining before the immunodetection. Experimental samples from the same sheep, and an internal standard consisting of the pre sample of one sheep, were loaded on the same SDS-PAGE. Protein signals were background-corrected, and related to the signal of the internal standard to reveal relative protein levels per total protein. Combined protein levels were calculated as the means of the relative values of the assessed proteins in each sample.

\section{Metabolomics and Lipidomics}

Nonpolar and polar compounds were extracted from $10 \mathrm{~mm}^{3}$ of biopsy material from infraspinatus muscle in cold methanol/ methyl tert-butyl ether/ $\mathrm{H}_{2} \mathrm{O} 360: 1200: 348 \mathrm{vol} / \mathrm{vol} / \mathrm{vol}$ using a full glass Potter-type homogenizer and subjected to ultrahighperformance liquid chromatography-tandem mass spectrometry essentially as described. ${ }^{13}$ The nonpolar phase and the polar phase, containing the majority of lipids and metabolites, respectively, were stored at $-30^{\circ} \mathrm{C}$ until being dried down and reconstituted for separation via ultra performance liquid chromatography. 
Lipids were separated with a $\mathrm{C}_{18}$ reversed-phase column (HSS T3; $1.7 \mathrm{~mm}$ external diameter, $0.2 \times 150 \mathrm{~mm}$ ) by a NanoAquity ultra performance liquid chromatography (Waters, Baden-Dättwil, Switzerland), with the application of a gradient of $5 \mu \mathrm{mol} / \mathrm{L}$ ammonium acetate in acetonitrile/water 40:60 vol $/ \mathrm{vol}(\mathrm{A})$ and $5 \mu \mathrm{mol} / \mathrm{L}$ ammonium acetate in acetonitrile/isopropanol 10:90 vol/vol. The eluate was injected at 6-second intervals into a Q Exactive Hybrid QuadrupoleOrbitrap Mass Spectrometer (Thermo Fisher Scientific, Reinach, Switzerland) by a nano-ESI source that was operated in positive-polarization mode. Metabolites were separated on the NanoAquity UPLC system (Waters) equipped with a BEH-Amide capillary column $(200 \mu \mathrm{m} \times 150 \mathrm{~mm}, 1.7-\mu \mathrm{m}$ particle size; Waters), applying a gradient of $0.5 \mu \mathrm{mol} / \mathrm{L}$ ammonium acetate in acetonitrile (A) and $0.5 \mu \mathrm{mol} / \mathrm{L}$ ammonium acetate in water (B) from $90 \%$ to $50 \%$ A and injected into a Q Exactive Hybrid Quadrupole-Orbitrap Mass Spectrometer. Mass spectrometry data were acquired using negative polarization and all ion fragmentation. All solvents used were of quality high-performance liquid chromatography grade (Chromasolv; Sigma-Aldrich, Buchs, Switzerland).

Three biological replicas were analyzed at the pre and post time points in the T2, T2-C, and T16 groups. All samples were injected as two technical replicates. Quality controls were run on individual and mixed samples for the determination of technical accuracy based on 20 selected compounds (amino acids, nucleotides, and metabolic intermediates) in mixed samples using a Quan Browser (Xcalibur software version 4.0; Thermo Fisher Scientific) and 61 further abundant ions using Progenesis QI software version 2.0 (Nonlinear Dynamics, Newcastle upon Tyne, UK, a Waters company). Mass spectrometry data sets were processed with Progenesis QI software to construct a consensus $\mathrm{m} / \mathrm{z}$ retention time map of detected compounds. L-carnitine, acetyl-L-carnitine, and palmitoylcarnitine were identified by individual inspection of the raw data files using the Qual Browser module in Xcalibur. For quantification of the identified L-carnitine and palmitoyl carnitine, the Quan Browser module of Xcalibur was used. Detected compound ions were further annotated by database searches (Lipid Maps, www.lipidmaps.org, last accessed January 6, 2020; Human Metabolome Data Base, www. $h m d b . c a$, last accessed January 6, 2020) based on derived neutral masses, isotopic patterns, and the match of observed fragmentation spectra and theoretical fragmentation spectra with a tolerance of 50 millidaltons. Data were normalized to the total signal per sample and subjected to statistical testing for the identification of compounds with demonstrated level alterations. Metabolomics data were deposited in the EMBL-EBI MetaboLights database (https://www.ebi.ac.uk metabolights/index, last accessed April 4, 2020) with the identifier MTBLS1490 (log-in required).

\section{Histologic Examination}

The area-percentages of muscle fiber types, lipid, and connective tissue were estimated, with quantitative microscopy of consecutive cryosections stained for slow- and fast-type myosin heavy chain, Goldner and Oil Red O staining, respectively. In brief, cryosections were prepared at $12-\mu \mathrm{m}$ thickness perpendicularly to the major axis of muscle fibers within the biopsy and stored at $-80^{\circ} \mathrm{C}$ until processed. Histologic Goldner and Oil Red O staining, respectively, were performed on thawed sections, with a standard protocol. ${ }^{13}$ The staining of slow- and fast-type myosin heavy chain was performed as described. ${ }^{38}$ In brief, the thawed sections were fixed in $4 \%$ paraformaldehyde, quenched with $0.3 \% \mathrm{H}_{2} \mathrm{O}_{2}$, and incubated after serial washes with phosphate-buffered saline in blocking solution of 5\% normal goat serum in phosphate-buffered saline. Subsequently, the sections were incubated with 1:100 dilutions of mouse antibody against slow-type myosin heavy chain (catalog number MAB1628; Millipore Corp., Temecula, CA) and rabbit antibody against fast-type myosin heavy chain (catalog number ab91506; Abcam, Cambridge, UK), followed by washes in phosphate-buffered saline and incubation with a 1:200 dilution of immunofluorescence-labeled secondary antibodies (anti-mouse Alexa Fluor 488, catalog number A11017; and anti-rabbit Alexa Fluor 555, catalog number A21428; Thermo Fisher Scientific). The stained sections were digitally recorded using an IX50 microscope with digital camera DP72 (Olympus, Wallisellen, Switzerland) that was operated with Cell Sens Dimension software version 1.6 (Olympus). Images from the Goldnerstained sections were analyzed using Adobe Photoshop CC version 20 (Adobe Systems Inc., Zurich, Switzerland) to reveal the area-percentage of muscle fibers (red structures) and connective tissue (green and nonstained structures). Lipid content and fiber type distribution were assessed in the images of the Oil Red O-stained and immunofluorescencestained sections, respectively, from the signal in the respective color channel using a macro with ImageJ software $^{40}$ version $1.48 \mathrm{v}$ (NIH, Bethesda, MD; http://imagej. nih.gov/ij). Numbers from the different fields were summed for the respective cellular structures to calculate the respective area-percentages of muscle fibers, lipid, and connective tissue for the entire cross section/biopsy. The percentage of lipid-free connective tissue was calculated as the difference between the respective area-percentage of connective tissue (as estimated from the Goldner staining) and lipid (as estimated in the Oil Red O staining).

\section{Radiologic Assessment of Structural Muscle Changes}

Computed tomography was conducted using a Somatom ART system (Siemens Medical Solutions, Erlangen, Germany) at a section thickness of $1 \mathrm{~mm}$. Magnetic resonance imaging was performed with a 3-Tesla system (Philips Ingenia 3T with dStream Body Coil Solution; Philips AG, Zurich, Switzerland) at a section thickness of $2 \mathrm{~mm}$. Both radiologic data sets were evaluated using an open-source DICOM viewer (OsiriX version 5.6, 32-bit; Pixmeo SARL, Bernex, Switzerland) from transverse sections of the 

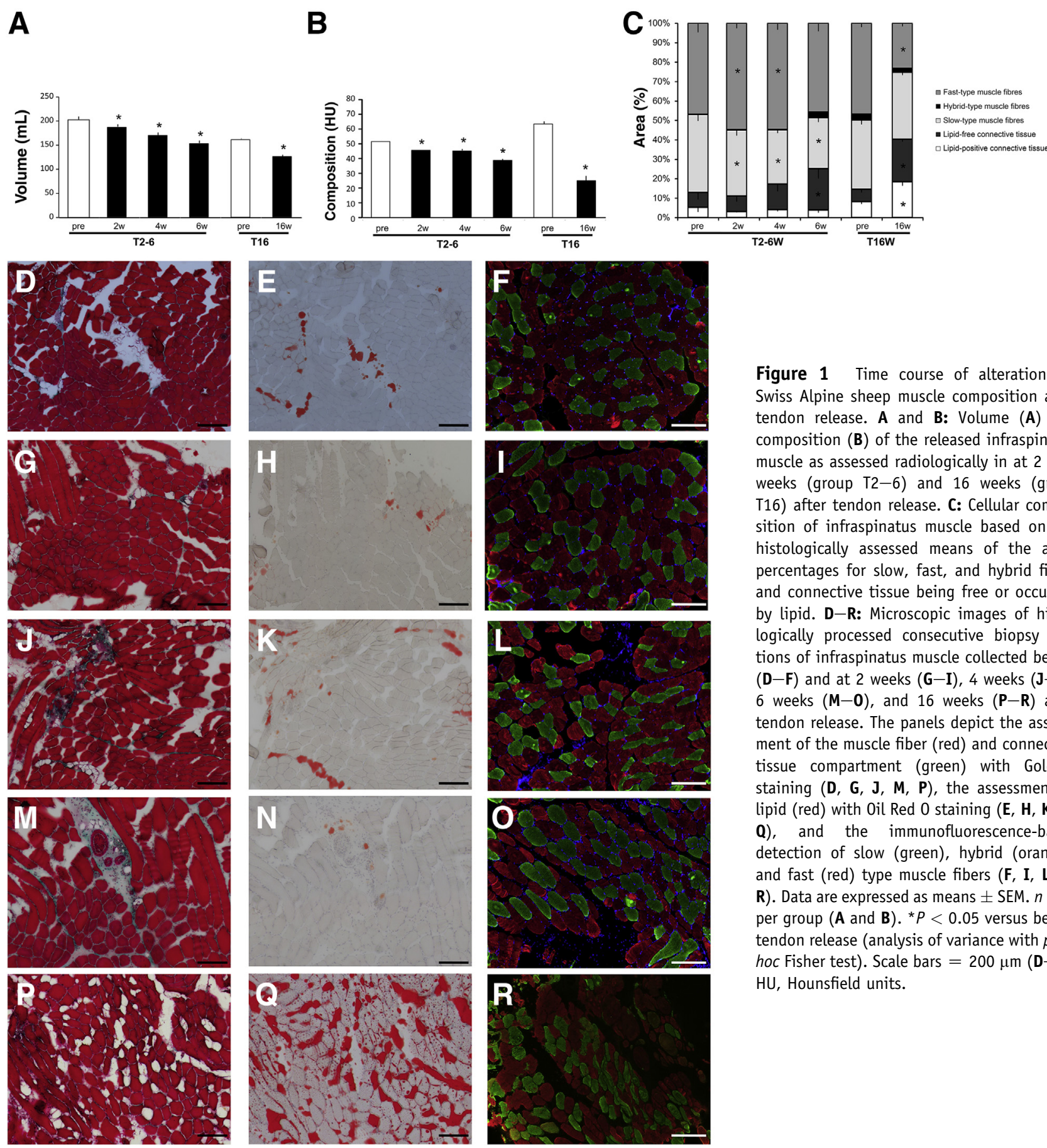

Figure 1 Time course of alterations in Swiss Alpine sheep muscle composition after tendon release. $\mathbf{A}$ and $\mathbf{B}$ : Volume $(\mathbf{A})$ and composition (B) of the released infraspinatus muscle as assessed radiologically in at 2 to 6 weeks (group T2-6) and 16 weeks (group T16) after tendon release. C: Cellular composition of infraspinatus muscle based on the histologically assessed means of the areapercentages for slow, fast, and hybrid fibers and connective tissue being free or occupied by lipid. D-R: Microscopic images of histologically processed consecutive biopsy sections of infraspinatus muscle collected before $(\mathbf{D}-\mathbf{F})$ and at 2 weeks $(\mathbf{G}-\mathbf{I}), 4$ weeks $(\mathbf{J}-\mathbf{L})$, 6 weeks $(\mathbf{M}-\mathbf{0})$, and 16 weeks $(\mathbf{P}-\mathbf{R})$ after tendon release. The panels depict the assessment of the muscle fiber (red) and connective tissue compartment (green) with Goldner staining $(\mathbf{D}, \mathbf{G}, \mathbf{J}, \mathbf{M}, \mathbf{P})$, the assessment of lipid (red) with Oil Red 0 staining $(\mathbf{E}, \mathbf{H}, \mathbf{K}, \mathbf{N}$, Q), and the immunofluorescence-based detection of slow (green), hybrid (orange), and fast (red) type muscle fibers (F, I, L, $\mathbf{0}$, R). Data are expressed as means \pm SEM. $n=6$ per group (A and $\mathbf{B})$. ${ }^{*} P<0.05$ versus before tendon release (analysis of variance with posthoc Fisher test). Scale bars $=200 \mu \mathrm{m}(\mathbf{D}-\mathbf{R})$. HU, Hounsfield units.

shoulders perpendicular to the glenoid cavity essentially as established. ${ }^{18,38}$ Magnetic resonance images were evaluated for the determination of fat fraction for the mean over the different muscle regions. Computed tomography scans were inspected for the retraction of infraspinatus muscle based on the distance of the bone chip respective to its original site of insertion, and segmented to reveal the density of muscle tissue from the mean over the different muscle regions. For the determination of muscle volume, the computed tomography sequences were imported into Osirix, and the area (ie, region of interest) being covered by infraspinatus muscle was outlined in each slice/segment with the closed polygon tool. Subsequently, the volume was calculated as the sum of the volumes as estimated in each slice/segment.

\section{Statistical Analysis}

Data on the assessed molecular species, that is, gene transcripts, metabolites, and lipids, were assessed using permutation-based statistics (ie, statistical analysis for 


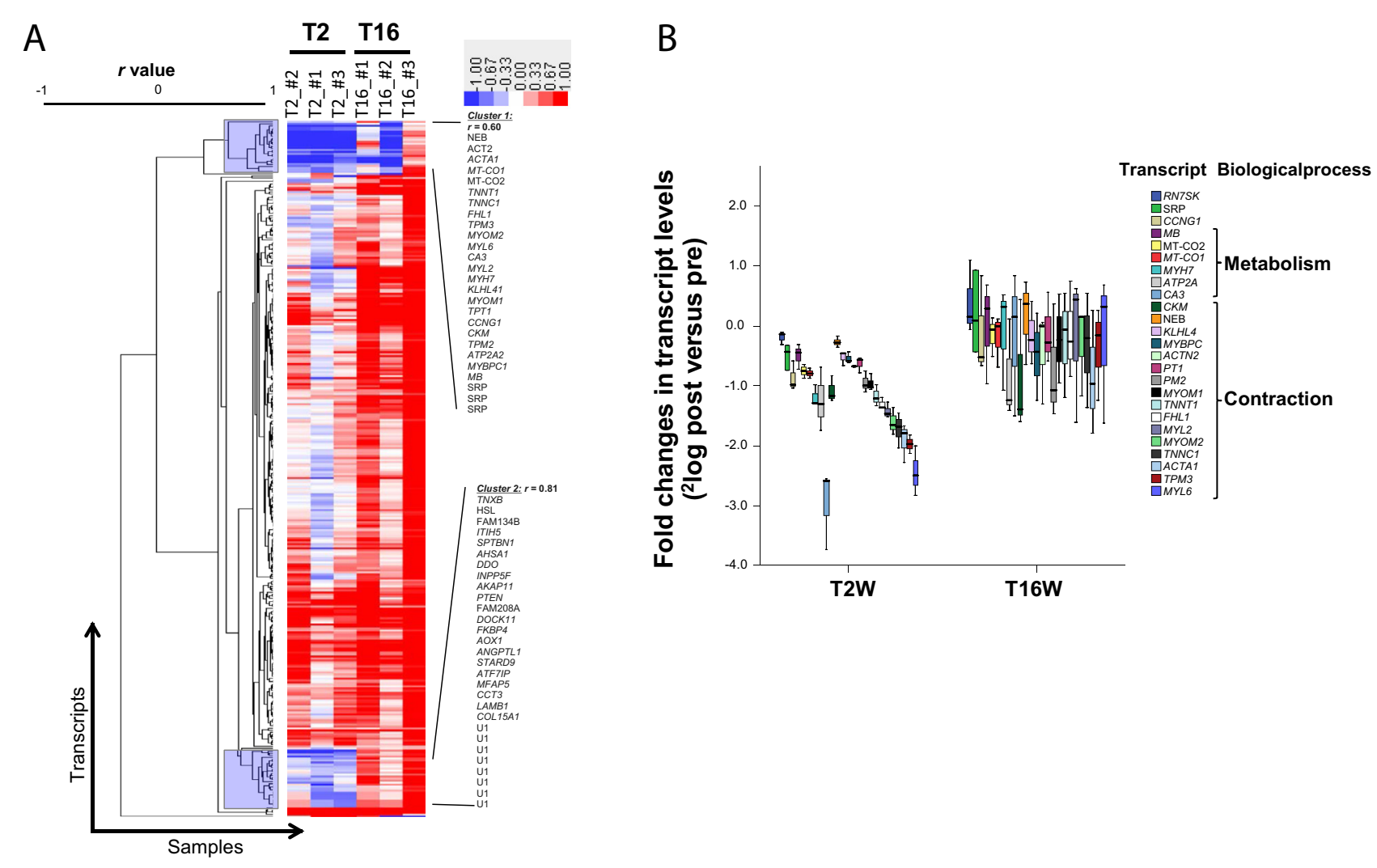

Figure 2 Transcriptome changes early and late into tendon release. A: Assembled heat map from the supervised cluster analysis of fold-level changes in the 348 gene transcripts that demonstrated trends ( $q$ value of $<10 \%$ ) for level alterations in infraspinatus muscle at 2 weeks (T2) or 16 weeks $(\mathrm{T} 16)$ after tendon release. Fold-changes are shown in color coding (red, increased; blue, decreased). Sample codes are indicated at the top. The dendrogram to the left indicates the level of correlation between the fold-changes. Two clusters with muscle-specific transcripts, which were down-regulated at 2 weeks after tendon release, are highlighted in blue on the dendrogram. The corresponding transcript abbreviations are given to the right of the heat map. B: Fold-changes in the expression levels in groups T2 and T16 for gene transcripts that demonstrated a significant down-regulation ( $q$ value of $<5 \%$ ) at 2 weeks after tendon release. Transcripts are arranged according to the biological process that describes their ontology. Data are expressed as medians (central lines), $95 \%$ CIs (boxes), and ranges (whiskers) (B). $n=3$ per group (A).

microarrays), on the basis of the values for signal intensity based on $q$ values, as described. ${ }^{13}$ Effects of tendon release on protein levels were assessed from the normalized pre and post values, with a repeated-measures analysis of variance with a post-hoc Fisher test for least significant differences using SPSS software version 23 (IBM, Armonk, NY). Global effects on the lipid levels per lipid class were assessed based on repeated-measures analysis of variances with a post-hoc Fisher test of the normalized lipid levels. A sign test was used for assessing the significance of postversus-pre differences in muscle volume and composition. Data are reported as means \pm SEM in the text, tables, and figures, and, where applicable, as medians (CIs) and ranges in whisker plots. Sample sizes are reported in the figure legends and tables, corresponding essentially to the numbers given in Design. The level of significance was set as $P<0.05$. The latter analyses were performed on the absolute values or after their referencing to pre values.

Co-regulation of molecular species, that is, gene transcripts, metabolites, and lipids, subjected to level alterations was assessed and visualized essentially as described by cluster analysis. ${ }^{13}$ For affected lipids, the significance of co-directional fold-changes per lipid class were also assessed based on a sign test. On instances, relative concentrations of the detected species were mean-centered respective to the pre values. Co-regulated transcripts were analyzed for the enrichment of gene ontologies using DAVID (https://david. ncifcrf.gov/summary.jsp, last accessed January 6, 2020). Altered metabolite or lipid species were assigned to a pathway based on the information provided in the HMDB entry (http://www.hmdb.ca, last accessed January 6, 2020) and MetaboAnalyst (http://www.metaboanalyst.ca/faces/ upload/PathUploadView.xhtml, last accessed January 6, 2020) or Lipid Maps (https://www.lipidmaps.org/datal classification/LM_classification_exp.php, last accessed January 6,2020 ).

\section{Results}

Time Course of Macro- and Microscopic Alterations in Rotator Cuff Muscle after Tendon Release

Supplemental Figure S1 depicts the different groups of animals used in the investigation. The changes in anatomic 
Table 1 Early Gene Transcript Alterations after Tendon Release

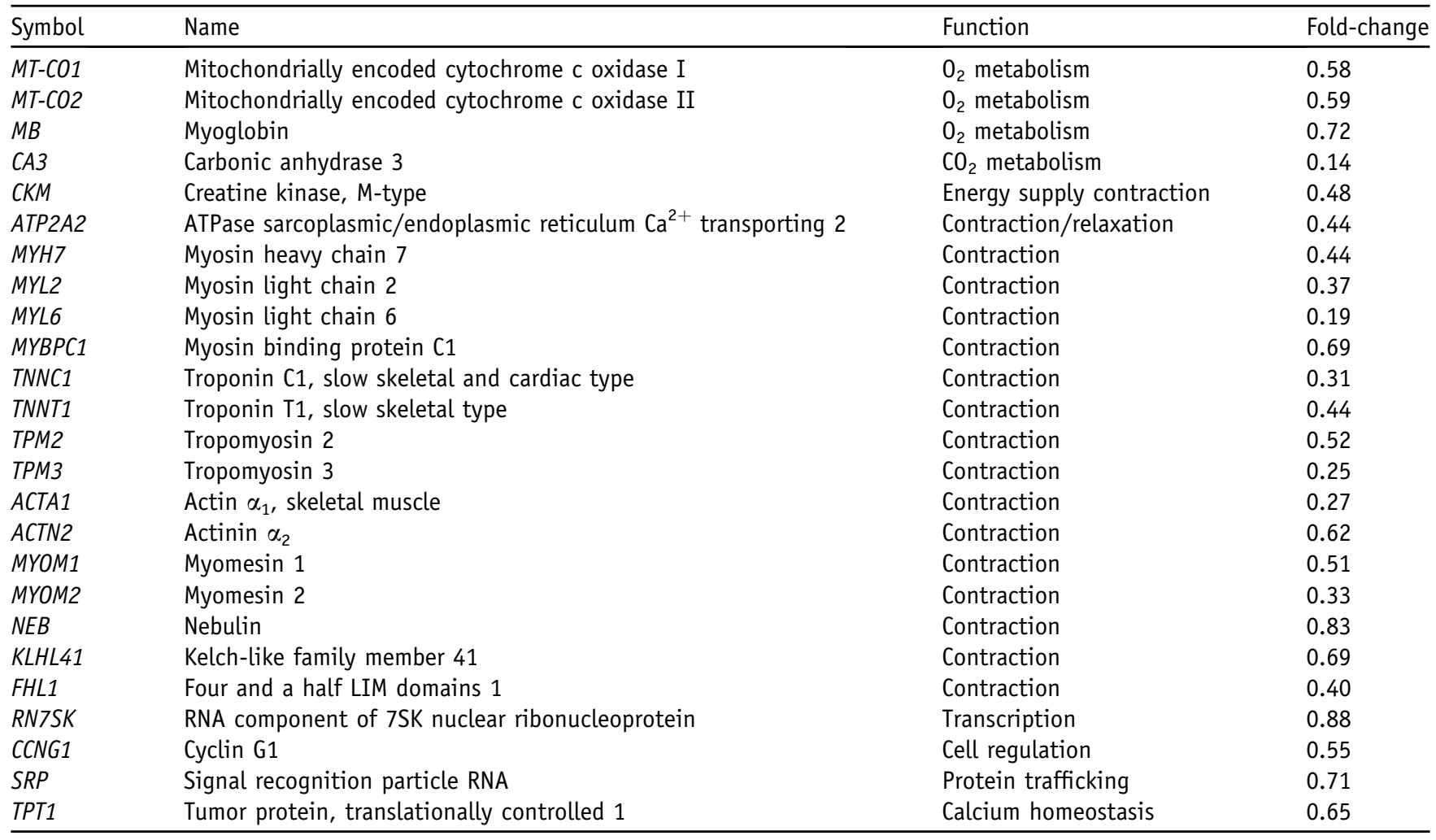

List of the gene transcripts, the corresponding gene ontologies, and fold-changes, being affected 2 weeks after tendon release. Statistical analysis for microarrays based on $q$ values of $<5 \%$.

hallmarks of sheep infraspinatus muscle over the first 16 weeks after tendon release are shown (Figure 1, A and B). Muscle volume was quantitatively lost starting at 2 weeks $(-9 \%)$ until 6 weeks $(-22 \%)$ after tendon release, and then stabilized until 16 weeks after tendon release. The composition of infraspinatus muscle was affected starting at 2 weeks after tendon release, based on lower Hounsfield units, which continued to fall until 16 weeks after tendon release.

The alterations in muscle composition, as determined microscopically from histologically processed muscle biopsy samples are shown (Figure 1, C-R). Respective to pre values, the area-percentage of lipid-positive connective tissue in infraspinatus muscle was first increased at 16 weeks after tendon release $(+124 \%)$, but was not affected at 2,4 , or 6 weeks after tendon release $(P=0.324,0.691$, and 0.612 , respectively). The area-percentage of lipid-free connective tissue was first increased at 6 weeks after tendon release $(+175 \%)$ and remained elevated 16 weeks after tendon release $(+245 \%)$. The area-percentage of slow-type muscle fibers in infraspinatus muscle was reduced between 4 $(-30 \%)$ and $6(-35 \%)$ weeks after tendon release. The areapercentage of fast-type fibers was increased by $17 \%$ at 2 and 4 weeks after tendon release and then inverted to a $50 \%$ reduction at 16 weeks after tendon release. Over all sample points, the histologically determined area-percentages of muscle fibers $\left(r=0.651, P=5.4 \times 10^{-7}\right)$, fast-type muscle fibers $(r=0.550, P=0.001)$, total connective tissue $\left(r=-0.651, P=5.4 \times 10^{-7}\right)$, lipid-free connective tissue $\left(r=-0.567, P=2.7 \times 10^{-5}\right)$, and lipid-positive connective tissue $(r=-0.430, P=0.002)$, but not the areapercentages of slow-type muscle fibers $(r=0.250$, $P=0.142$ ), were correlated to the Hounsfield unit-based estimates of muscle composition.

\section{Generalized Down-Regulation of the Slow 0xidative Gene Program}

The contribution of altered gene-transcript expression to the changes in infraspinatus muscle composition 2 weeks after tendon release was characterized in a new group T2, respective to those seen after 16 weeks of tendon release (group T16). At 2 weeks after tendon release, the muscles in group T2 had lost $9 \%$ of their volume $(185.7 \pm 9.6$ versus $168.7 \pm 8.6 \mathrm{~mL}$; $P=0.0004 ; n=6$ ) and demonstrated an altered composition $(66.1 \pm 2.9$ versus $53.3 \pm 1.3$ Hounsfield units, $P=0.0082)$. The concentration of RNA per muscle volume was not affected at 2 weeks after tendon release (371.5 \pm 31.5 versus $354.4 \pm 12.5 \mathrm{ng} / \mathrm{mm}^{3} ; P=0.643$; group T2) and 16 weeks after tendon release $\left(309.7 \pm 85.7\right.$ versus $358.5 \pm 20.4 \mathrm{ng} / \mathrm{mm}^{3}$; $P=0.536$; group T16).

Expressed gene transcripts were detected by RNA sequencing, yielding a total of 12,173 annotated gene 
transcripts. The pattern of the response for the relative concentration of 348 transcripts being affected either 2 or 16 weeks after tendon release is shown (Figure 2A). Compared to the respective pre values, the levels of 25 gene transcripts in infraspinatus muscle were altered 2 weeks after tendon release, all of them lowered (Figure 2B).
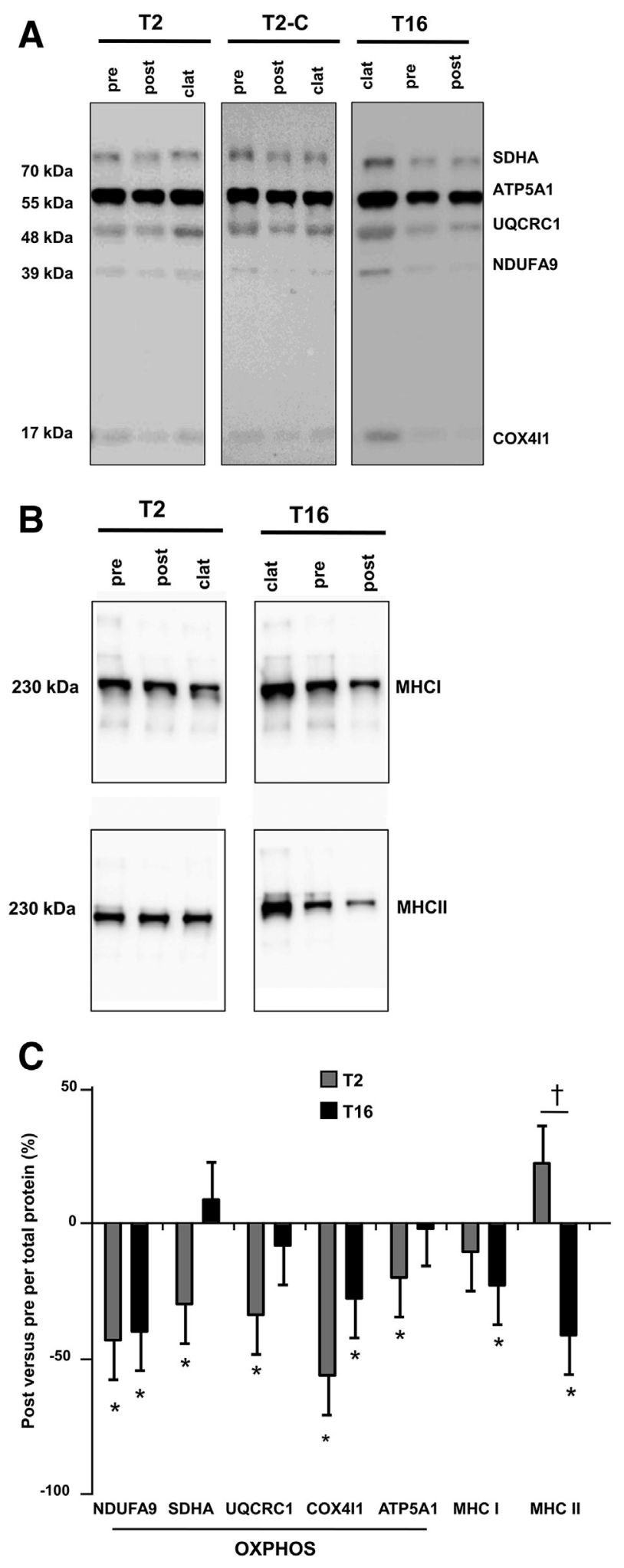

Seventeen of the affected gene transcripts were associated with contraction, 4 were associated with mitochondrial oxygen or carbon dioxide metabolism, and 4 , with aspects of cell regulation (Table 1). The former set of 25 gene transcripts were not affected at 16 weeks after tendon release. Gene ontologies of altered transcripts associated with lipid biosynthesis, oxidation, and adipocyte differentiation markers were not significantly affected at 2 and 16 weeks after tendon release. In contrast, transcripts of gene ontologies associated with cellular components focal adhesions (32), extracellular exosomes (77), extracellular space (39), and calcium binding (26) were prominently increased at 16 weeks after tendon release (Supplemental Table S1).

Time Course of Level Alterations in Mitochondrial and Contractile Protein

Level alterations of protein constituents from the five complexes of mitochondrial respiration (NDUFA9, SDHA, UQCRC1, COX4I1, and ATP5A1), and myosin heavy chain, were assessed (Figure 3, A and B). The combined levels of mitochondrial proteins were reduced at 2 $(27 \% \pm 19 \%), 4(30 \% \pm 14 \%)$, and $6(20 \% \pm 19 \%)$ weeks after tendon release, but they were not affected at 16 weeks after tendon release $(-9 \%, P=0.390)$. Compared to the pre levels, NDUFA9 $(-44 \%)$, SDHA $(-30 \%)$, UQCRC1 $(-34 \%)$, and COX4I1 $(-57 \%)$ were lower at 2 weeks after tendon release (Figure 3C). At 16 weeks after tendon release, the levels of NDUFA9 and COX4I1 protein were reduced ( $-40 \%$ and $-28 \%$, respectively), compared to the pre values. Protein level of fast-type myosin heavy chain showed a trend for an increase at 2 weeks after tendon release $(+23 \%, P=0.10)$ and a reduction at 16 weeks after tendon release $(-34 \%)$. The changes in fast-type myosin heavy chain levels differed between 2 and 16 weeks after tendon release. The protein levels of slow-type myosin heavy chain demonstrated a trend toward a reduction at 16 weeks after tendon release $(-23 \%, P=0.090)$.

Figure 3 Expression of mitochondrial protein. $\mathbf{A}$ and $\mathbf{B}$ : Representative images of the detected protein constituents of mitochondrial respiration $(\mathbf{A})$ and slow-type (MHCI) and fast-type (MHCII) myosin heavy chains (B) in infraspinatus muscle of one sheep before (pre) and 2 weeks after (post) tendon release with (group T2-C) or without (group T2) L-carnitine administration, and 16 weeks after tendon release (group T16), as well as the respective contralateral controls (clat). Ten micrograms of total protein was loaded per lane. Blots being separated with a frame stem from different experiments. Bottom, Ponceau $\mathrm{S}-$ stained actin bands of the blotted membrane showing equal loading. $\mathrm{C}$ : Percentage changes in the levels of the five proteins of mitochondrial respiration (OXPHOS) and slow- and fast-type myosin heavy chain (per total protein) in infraspinatus muscle in groups T2 and T16. Data are expressed as means \pm SEM. $n=6$ per group. ${ }^{*} P<0.05$ versus before tendon release (analysis of varianc with post-hoc Fisher test); ${ }^{\dagger} P<0.05$ (analysis of variance with post-hoc Fisher test). ATP5A1, ATP synthase F1 subunit $\alpha$; COX4I1, cytochrome $c$ oxidase subunit 4I1; NDUFA9, NADH:ubiquinone oxidoreductase subunit A9; SDHA, succinate dehydrogenase complex flavoprotein subunit A; UQCRC1, ubiquinol-cytochrome $C$ reductase core protein 1 . 


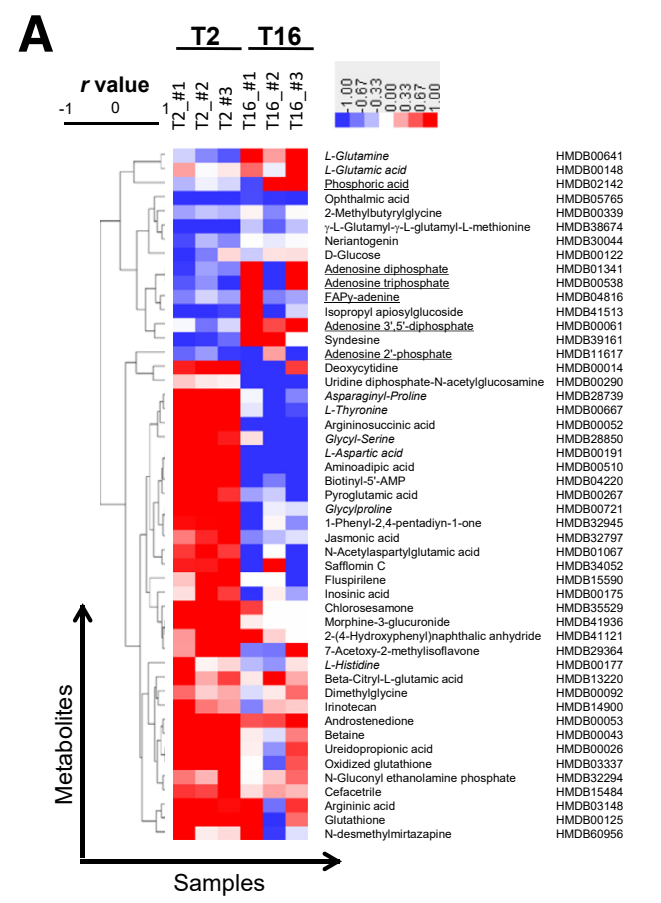

\section{B}
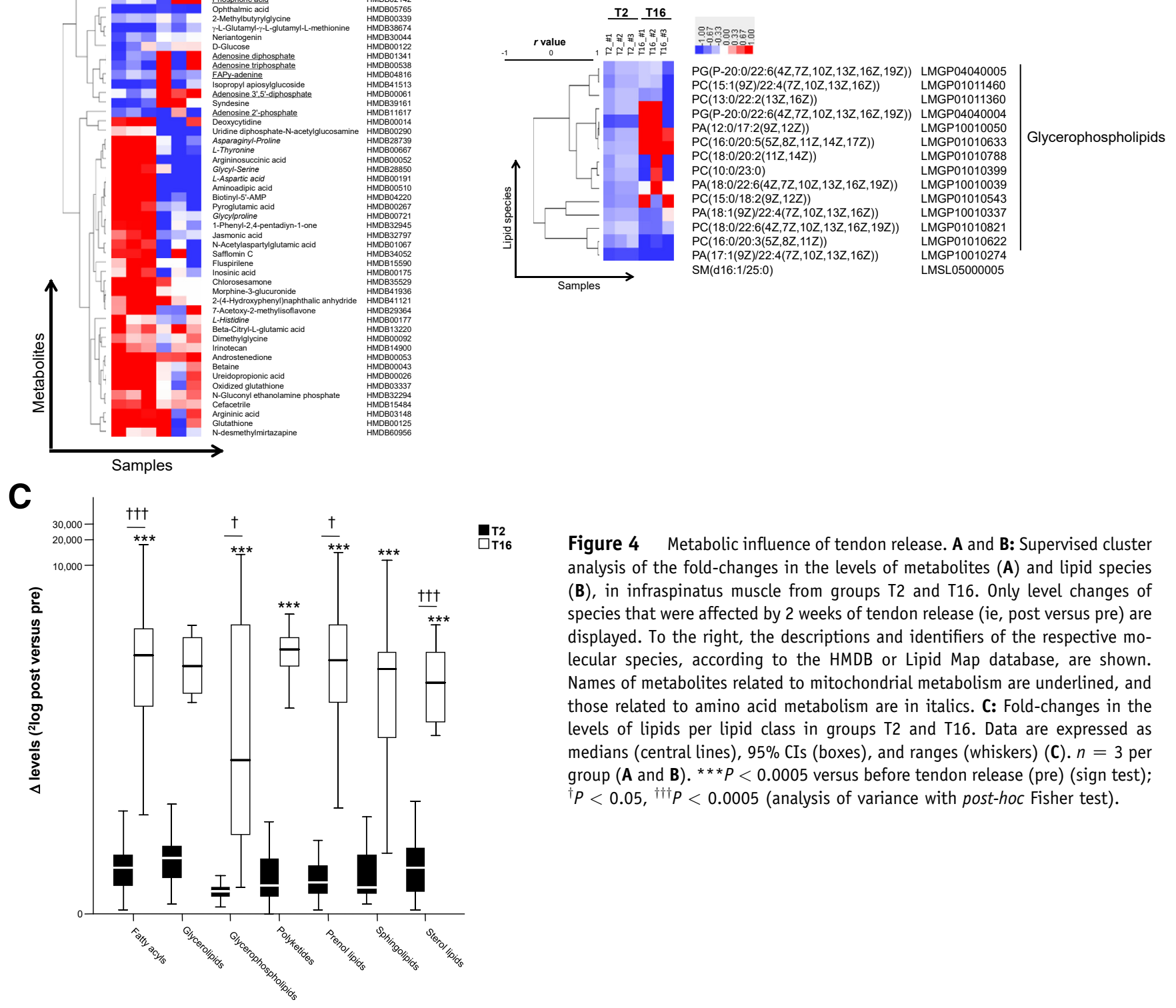

Figure 4 Metabolic influence of tendon release. A and B: Supervised cluster analysis of the fold-changes in the levels of metabolites (A) and lipid species (B), in infraspinatus muscle from groups T2 and T16. Only level changes of species that were affected by 2 weeks of tendon release (ie, post versus pre) are displayed. To the right, the descriptions and identifiers of the respective molecular species, according to the HMDB or Lipid Map database, are shown. Names of metabolites related to mitochondrial metabolism are underlined, and those related to amino acid metabolism are in italics. C: Fold-changes in the levels of lipids per lipid class in groups T2 and T16. Data are expressed as medians (central lines), $95 \%$ CIs (boxes), and ranges (whiskers) (C). $n=3$ per group (A and $\mathbf{B}) .{ }^{* * *} P<0.0005$ versus before tendon release (pre) (sign test); ${ }^{\dagger} P<0.05,{ }^{\dagger \dagger} P<0.0005$ (analysis of variance with post-hoc Fisher test).

\section{Metabolomic Effects of Tendon Release}

Totals of 717 metabolites and 1068 lipid compounds in infraspinatus muscle were detected and mapped in groups $\mathrm{T} 2$ and $\mathrm{T} 16$, that is, at 2 and 16 weeks after tendon release. The respectively defined metabolome and the lipidome demonstrated distinct differences between the released muscle and its contralateral control (Figure 4).

At 2 weeks after tendon release, the levels of 49 metabolites in infraspinatus muscle were affected in the released muscle compared to its contralateral control (Figure 4A and Supplemental Table S2). The main themes

of the metabolomic alterations at 2 weeks after tendon release were that the levels of 12 metabolites associated with amino acid metabolism were increased in the released muscle compared to the contralateral control, when the levels of four nucleosides associated with oxidative phosphorylation were lowered, and one resulting from their deamination, that is, inosinic acid, was increased. The levels of 5 metabolites associated with pyrimidine metabolism, and 3 metabolites associated with glutathione metabolism, were increased in released muscle. Six metabolites associated with lipid metabolism were affected as well. 


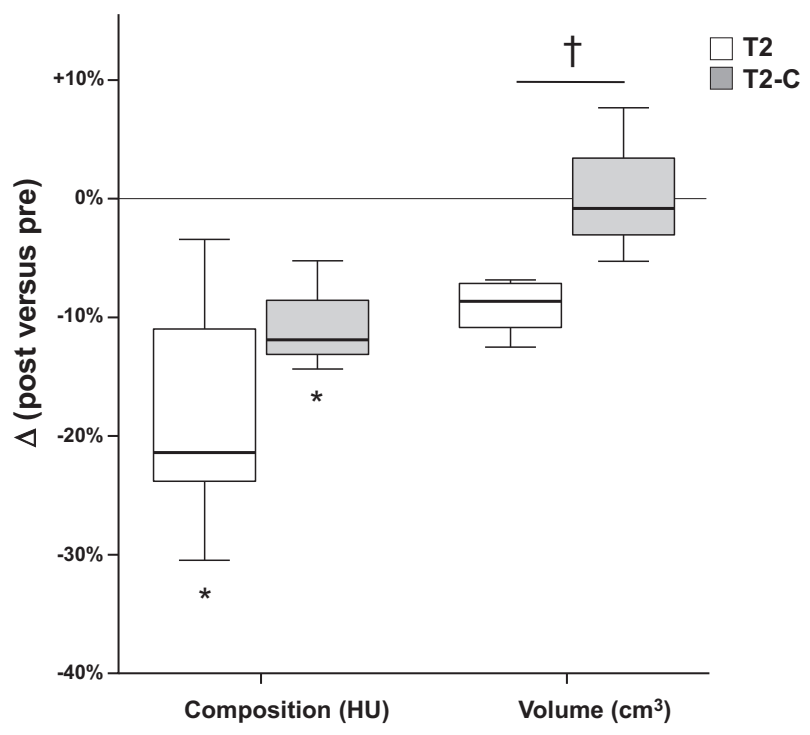

Figure 5 Anatomic effects of L-carnitine administration with tendon release. Differences in the volume and composition of infraspinatus muscle in the L-carnitine-treated (T2-C) and -untreated (T2) groups from before to after tendon release (post versus pre). Data are expressed as medians (central lines), $95 \%$ CIs (boxes), and ranges (whiskers). $n=3$ (T2-C); $n=6$ (T2). ${ }^{*} P<0.05$ versus before tendon release (sign test) ${ }^{\dagger} P<0.05$ (analysis of variance with post-hoc Fisher test). HU, Hounsfield units.

For the lipidome, the levels of 14 glycerophospholipids and one saccharolipid, were reduced at 2 weeks after tendon release (Figure 4B and Supplemental Table S3). At 16 weeks after tendon release, the levels of lipids in classes representing fatty acyls, glycerophospholipids, prenol lipids, and sterol lipids were strongly increased, differing from the alterations at 2 weeks after tendon release (Figure 4C). The level of L-carnitine was increased in the released muscles (Supplemental Table S4).

\section{Structural Effects of L-Carnitine Administration in the First 2 Weeks after Tendon Release}

The administration of L-carnitine was associated with a reduction in infraspinatus muscle volume at 2 weeks after tendon release compared to that in the sheep that did not receive L-carnitine $(0.5 \% \pm 3.8 \%$ versus $-9.1 \% \pm 0.9 \%$, $P=0.0120$ ) (Figure 5). Based on Hounsfield units, the alteration in infraspinatus muscle composition at 2 weeks after tendon release was not affected by L-carnitine administration $(P=0.228)$ (Figure 5).

\section{Altered Mitochondrial Protein with L-Carnitine Administration}

In the infraspinatus muscle of the sheep in group T2-C, which received L-carnitine in the week prior to tendon release, the pre level of NDUFA9 was $106 \%$ higher than that in the sheep that did not receive L-carnitine (Figure 6, A and B). With L-carnitine treatment, the tentative lowering of the ATP5A1 protein level at 2 weeks after tendon release $(-21 \%, P=0.098)$ was prevented $(-2 \%, P=0.90)$ (Figure 6C).

\section{Metabolic Effects of L-Carnitine Administration}

The levels of L-carnitine and 28 acyl-carnitines in infraspinatus muscle were lower at 2 weeks after tendon release in the L-carnitine-treated sheep compared to the untreated sheep (Supplemental Table S4); when the levels of 2 acyl-carnitines were elevated. In the contralateral muscle, the levels of 4 acyl-carnitines were higher in the L-carnitine-treated compared to the untreated sheep, whereas the levels of 3 acyl-carnitines were lower.

Respective to the lipidome, L-carnitine administration was associated with affected levels of a large portion of lipids in a way opposite of that in the contralateral muscle (Figure 7A). Globally lower lipid levels were identified in released muscle for the lipid classes of fatty acyls, glycerolipids, and sphingolipids with L-carnitine administration (Figure 7C). In contrast, the levels of glycerophospholipids in released muscle were higher in the L-carnitine-treated sheep (Supplemental Table S5) (including nine species that recovered from the lowered muscle levels in untreated sheep) (Table 2). L-Carnitine-induced alterations also comprised an increased saturation level of carbon bonds and the carbon-chain length of a number of glycerophospholipids and glycerolipids (Supplemental Table S5). L-Carnitine-induced alterations also comprised an increased saturation level of carbon bonds and the carbonchain length of a number of glycerophospholipids and glycerolipids.

The response of the metabolome to L-carnitine administration was qualitatively similar in released and contralateral infraspinatus muscle despite quantitative differences in levels of 35 metabolites between released and contralateral muscle with L-carnitine administration (Figure 7B and Supplemental Table S6). L-Carnitine administration did partially correct the levels of 8 metabolites, which were affected at 2 weeks after tendon release toward the respective pre levels (Table 3). Two of the metabolites (ie, Lglutamic acid and dimethylglycine) were associated with the class of amino acids, and one (2-methylbutyrylglycine), with mitochondrial $\beta$-oxidation. In contrast, nucleosides associated with high-energy reserves through oxidative phosphorylation, that is, ATP, adenosine $5^{\prime}$-diphosphate, adenosine $2^{\prime}$-phosphate, and phosphocreatine, were further lowered with L-carnitine administration in the released muscle when the related inosinic acid was increased. The alterations with L-carnitine administration comprised an increased level of the intermediate of lipid hydrolysis, glycerol 3-phosphate, as well as a tendinous reduction in the $\beta$-oxidation-related $\mathrm{NAD}^{+}$level in released infraspinatus muscle $(-38 \%, P=0.053)$ and its contralateral control $(-39 \%, P=0.087)$. Conversely, the abundance of its 
A

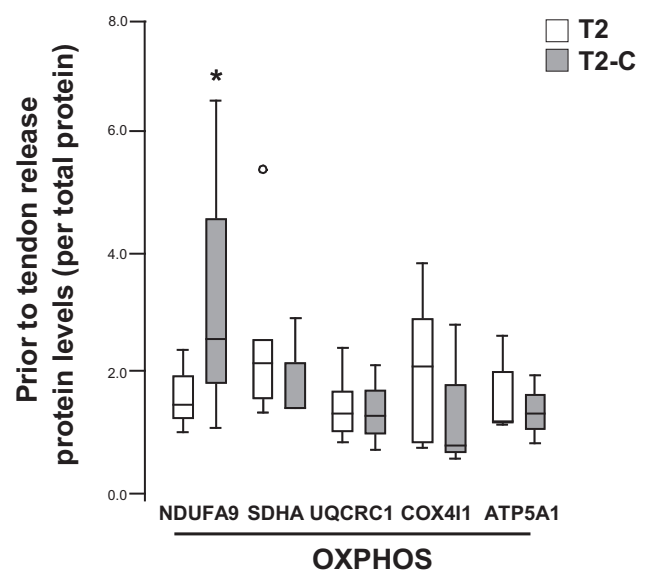

B

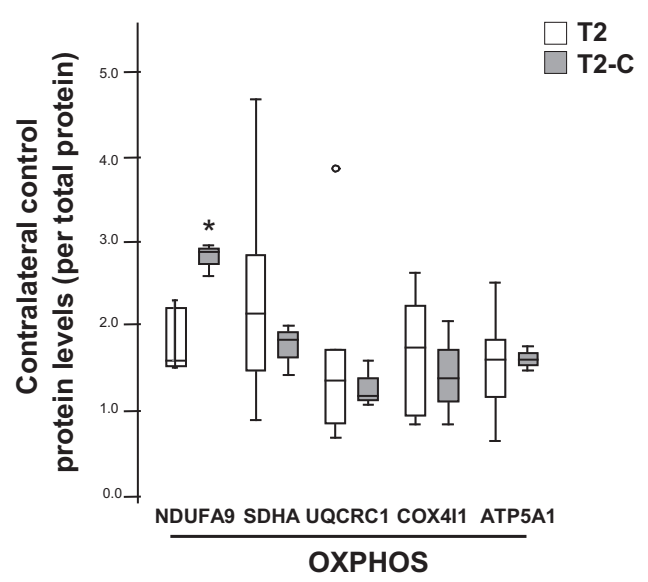

C

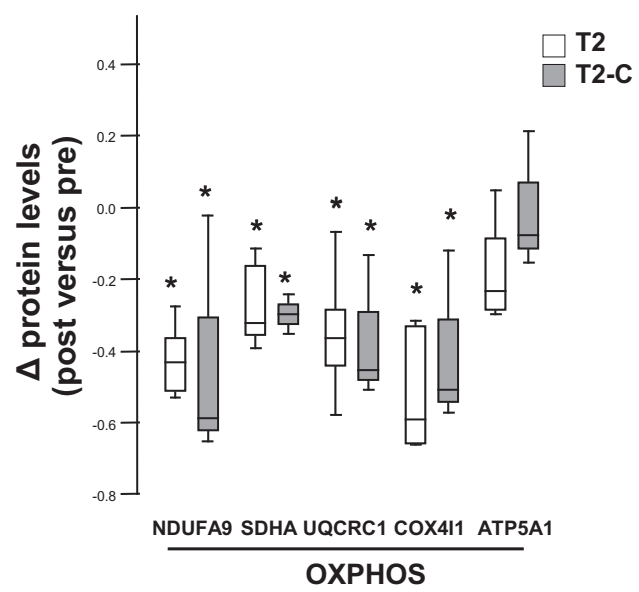

Figure 6 Influence of L-carnitine administration on respiratory chain constituents with tendon release. A and B: Protein constituents of mitochondrial respiration (OXPHOS) in infraspinatus muscle prior to (A) and 2 weeks after tendon release in the contralateral controls (B) in sheep treated with Lcarnitine (T2-C) or not (T2). C: Changes in levels of the mitochondrial protein 2 weeks after tendon release versus pre levels in group T2-C and T2. L-Carnitine administration started 1 week prior to tendon release. Data are expressed as medians (central lines), $95 \%$ CIs (boxes), and ranges (whiskers). $n=3$ (T2-C); $n=6$ (T2). ${ }^{*} P<0.05$ T2 versus T2-C (analysis of variance with post-hoc Fisher test). ATP5A1, ATP synthase F1 subunit $\alpha$; COX4I1, cytochrome $c$ oxidase subunit 4I1; NDUFA9, NADH:ubiquinone oxidoreductase subunit A9; SDHA, succinate dehydrogenase complex flavoprotein subunit A; UQCRC1, ubiquinolcytochrome $\mathrm{c}$ reductase core protein 1. reduced form, NADH, was not affected $(P=0.119$ and $0.248)$.

\section{Discussion}

The accumulation of fat and the loss of contractile material are hallmarks of many degenerative muscle diseases, including dystrophy and rotator cuff disease. ${ }^{5-7}$ Here we explored the course of alterations in the cellular composition of rotator cuff muscle in sheep during the first 16 weeks after tendon release, and assessed molecular composition at selected time points, in order to identify metabolic processes that are associated with the degradation of muscle fibers and lipid accumulation. ${ }^{13,15,38}$

Our multilevel characterization exposed that atrophy and lipid accumulation in sheep infraspinatus muscle after tendon release took place in two different phases. Atrophy was essentially confined to the first 6 weeks after tendon release and was related to a slow-to-fast fiber shift of muscle composition that became manifest as early as 2 weeks after tendon release. These myocellular alterations were reflected by the specific down-regulation of oxygen metabolism and slow contraction-associated gene expression, reductions in oxidative phosphorylation-associated nucleosides and glycerophospholipids, as well as increased levels of alanine, aspartate, and glutamate metabolism-related amino acids. Histologically, lipid accumulated between 6 and 16 weeks after tendon release, subsequent to an increase in the areapercentage of lipid-free connective tissue, and in correspondence to a reduction in the area-percentage of fast-type muscle fibers. The oral administration of L-carnitine prevented muscle atrophy (Figure 5) and mitigated perturbations in the levels of nine glycerophospholipids (Table 2) and the main amino acid component of skeletal muscle, glutamic acid (Table 3), during the first 2 weeks after tendon release. However, L-carnitine did not correct the abundance of mitochondria-associated proteins (except for a tentative effect on ATP5A1) (Supplemental Table S6) and nucleosides (Table 3).

Considerable linear relationships were identified between the computed tomography-based measure of muscle composition in Hounsfield units, and histologic measures of muscle composition during its response to tendon release. This observation supports the general validity of relying on radiologic measures to draw conclusions on the cellular degeneration of rotator cuff muscle after tenotomy. However, whereas correlations were noted for the reciprocal area-percentages of muscle fibers and total connective tissue (ie, $r=0.651$ and $r=-0.651$, respectively), lipid-positive connective tissue and fast-type muscle fibers (ie, $r=-0.430$ and $r=0.550$, respectively); the area-percentage of slow-type muscle fibers was not correlated with the Hounsfield unit $(r=0.250$, $P=0.142$ ). This observation advocates a certain level of caution when interpreting average values from radiologic measurements regarding the early degenerative changes of rotator cuff muscle composition after tendon release. 
A

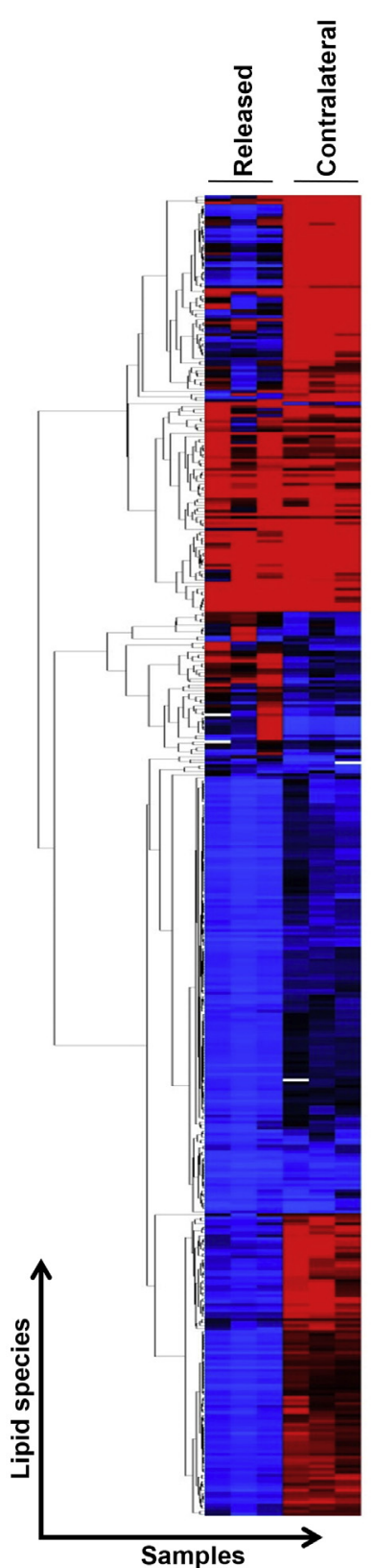

B

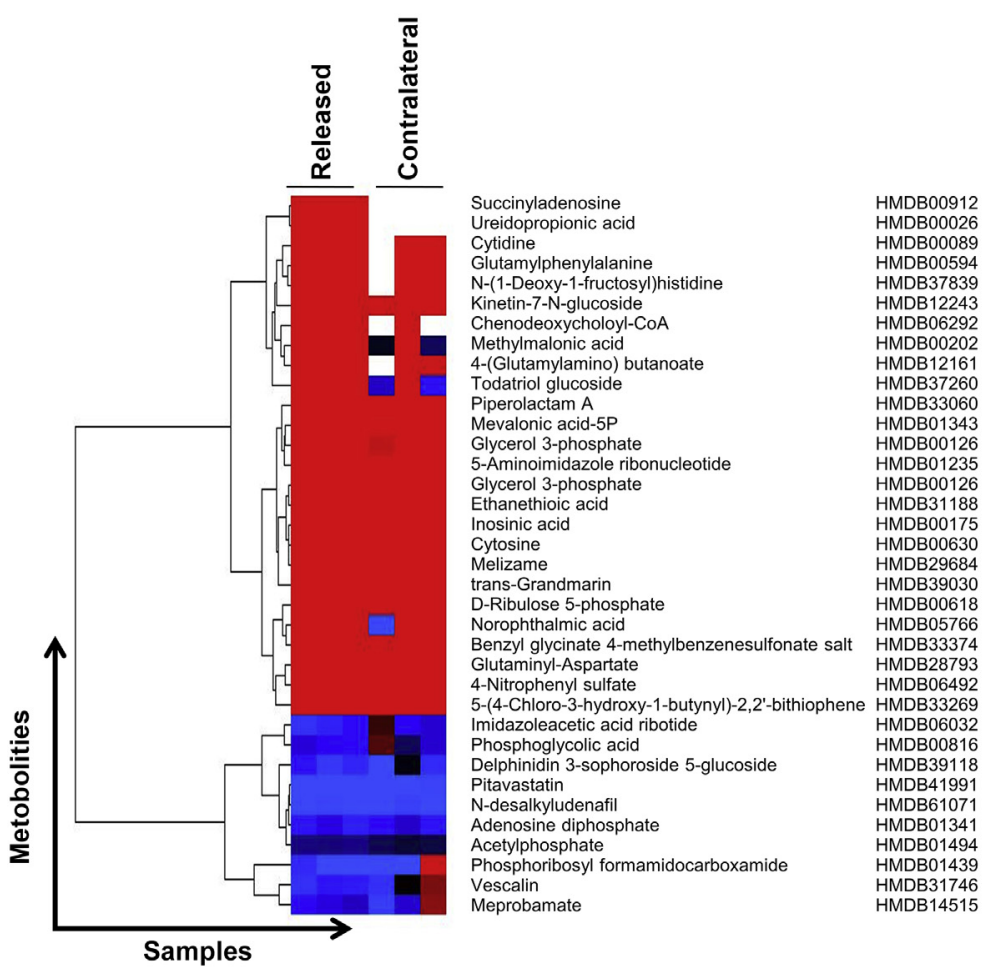

\section{C}

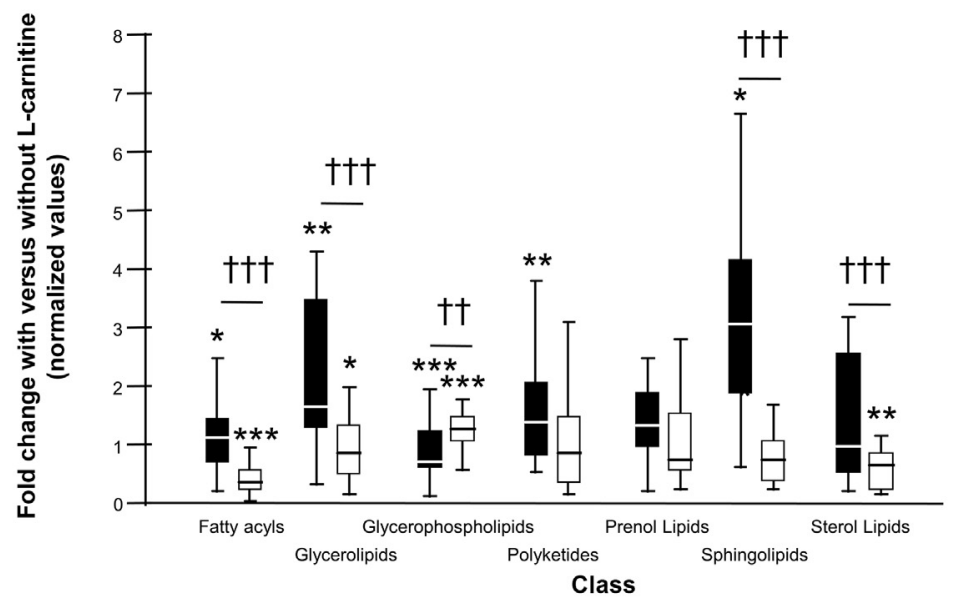

Figure 7 Tendon release modifies the influence of L-carnitine on the muscle lipidome. A and B: Supervised cluster analysis of fold-changes post versus pre in 441 lipid species (A) and 35 metabolites (B) in the L-carnitine-treated group in infraspinatus muscle at 2 weeks after release and its contralateral control. A: Only species affected by L-carnitine administration in released muscle and its contralateral control are displayed. B: Only species affected by L-carnitine administration in released muscle that differed from the level alterations in contralateral muscle are considered ( $q$ value of $<5 \%$ ). To the right, the identifiers in the Lipid Maps and HMDB databases are shown. C: Fold-changes in the levels of lipid per lipid class in groups T2 and T2-C. Data are expressed as medians (central lines), $95 \%$ CIs (boxes), and ranges (whiskers) (C). $n=3$ (A and B). ${ }^{*} P<0.05,{ }^{*} P<0.005$, and ${ }^{* \star *} P<0.0005$ versus without $\mathrm{L}-\mathrm{carnitine}$ (sign test) ${ }^{\dagger \dagger} P<0.005,{ }^{\dagger \dagger} P<0.0005$ (analysis of variance with post-hoc Fisher test).

Mitochondrial dysfunction has been pointed out to contribute to muscle atrophy ${ }^{20,21}$ and the accumulation of lipid in skeletal muscle. ${ }^{22}$ For instance, in models of muscle disuse, the down-regulation of mitochondrial capacity via the (transient) reduction in mitochondrial transcript and protein expression ${ }^{23,24,27,41}$ may modulate both the synthesis and breakdown of fatty acids. ${ }^{42,43}$ Recently it was pointed out in a rat model that a reduction in the functional capacity of mitochondria to metabolize organic substrates, including fatty acids, likely leads to lipid accumulation with rotator cuff injury. ${ }^{36}$ In the present study in a sheep model of rotator cuff muscle degeneration, a specific down-regulation of mitochondrial proteins and transcripts, and reduced levels of oxidative phosphorylation-associated nucleosides were 
Table 2 A Sheep Model Lipid Species Being Affected by 2 Weeks of Tendon Release and L-Carnitine Administration

\begin{tabular}{|c|c|c|c|c|c|c|c|c|c|c|}
\hline \multirow[b]{2}{*}{ Description } & \multirow[b]{2}{*}{ Identifier } & \multirow[b]{2}{*}{ Formula } & \multirow[b]{2}{*}{ Substance class } & \multirow[b]{2}{*}{ Location } & \multicolumn{2}{|c|}{$\begin{array}{l}\text { Released versus } \\
\text { contralateral }\end{array}$} & \multicolumn{2}{|c|}{$\begin{array}{l}\text { L-Carnitine versus } \\
\text { no L-carnitine } \\
\text { (released) }\end{array}$} & \multicolumn{2}{|c|}{$\begin{array}{l}\text { L-Carnitine versus } \\
\text { no L-carnitine } \\
\text { (contralateral) } \\
\end{array}$} \\
\hline & & & & & $\begin{array}{l}\text { Fold- } \\
\text { difference }\end{array}$ & $\begin{array}{l}q \\
\text { Value, } \\
\%\end{array}$ & $\begin{array}{l}\text { Fold- } \\
\text { difference }\end{array}$ & $\begin{array}{l}q \\
\text { Value, } \\
\%\end{array}$ & $\begin{array}{l}\text { Fold- } \\
\text { difference }\end{array}$ & $\begin{array}{l}q \\
\text { Value, } \\
\%\end{array}$ \\
\hline $\begin{array}{l}\text { 1-[8-(5)-ladderane-octanyl]-2- } \\
\text { [8-(3)-ladderane-octanyl]- } \\
\text { sn-glycero-3-phospho- } \\
\text { (1'-sn-glycerol) }\end{array}$ & LMGP04040004 & $\mathrm{C}_{46} \mathrm{H}_{77} \mathrm{O}_{8} \mathrm{P}$ & Glycerophospholipids & $\begin{array}{l}\text { Plasma } \\
\text { membrane }\end{array}$ & 0.59 & 0.00 & 0.70 & 0.00 & & \\
\hline$\underline{P A}[12: 0 / 17: 2(9 Z, 12 Z)]$ & LMGP10010050 & $\mathrm{C}_{32} \mathrm{H}_{59} \mathrm{O}_{8} \mathrm{P}$ & Glycerophospholipids & $\begin{array}{l}\text { Plasma } \\
\text { membrane }\end{array}$ & 0.27 & 0.00 & 1.10 & 2.33 & 0.17 & 0.00 \\
\hline PA[17:1 (9Z)/22:4 (7Z,10Z,13Z,16Z)] & LMGP10010274 & $\mathrm{C}_{42} \mathrm{H}_{73} \mathrm{O}_{8} \mathrm{P}$ & Glycerophospholipids & $\begin{array}{l}\text { Plasma } \\
\text { membrane }\end{array}$ & 0.50 & 0.00 & 1.27 & 2.33 & 0.75 & 0.00 \\
\hline $\begin{array}{l}\mathrm{PA}[18: 0 / 22: 6 \\
\quad(4 Z, 7 Z, 10 Z, 13 Z, 16 Z, 19 Z)]\end{array}$ & LMGP10010039 & $\mathrm{C}_{43} \mathrm{H}_{73} \mathrm{O}_{8} \mathrm{P}$ & Glycerophospholipids & $\begin{array}{l}\text { Plasma } \\
\text { membrane }\end{array}$ & 0.67 & 0.00 & & & 0.74 & 0.00 \\
\hline$\underline{P A[18: 1(9 Z) / 22: 4(7 Z, 10 Z, 13 Z, 16 Z)]}$ & LMGP10010337 & $\mathrm{C}_{43} \mathrm{H}_{75} \mathrm{O}_{8} \mathrm{P}$ & Glycerophospholipids & $\begin{array}{l}\text { Plasma } \\
\text { membrane }\end{array}$ & 0.53 & 0.00 & 1.22 & 2.33 & & \\
\hline$\underline{P C(10: 0 / 23: 0)}$ & LMGP01010399 & $\mathrm{C}_{41} \mathrm{H}_{82} \mathrm{NO}_{8} \mathrm{P}$ & Glycerophospholipids & $\begin{array}{l}\text { Plasma } \\
\text { membrane }\end{array}$ & 0.51 & 0.00 & 1.24 & 2.33 & 0.73 & 0.00 \\
\hline$\underline{P C[13: 0 / 22: 2(13 Z, 16 Z)]}$ & LMGP01011360 & $\mathrm{C}_{43} \mathrm{H}_{82} \mathrm{NO}_{8} \mathrm{P}$ & Glycerophospholipids & $\begin{array}{l}\text { Plasma } \\
\text { membrane }\end{array}$ & 0.62 & 0.00 & 1.40 & 2.33 & & \\
\hline$\underline{P C}[15: 0 / 18: 2(9 Z, 12 Z)]$ & LMGP01010543 & $\mathrm{C}_{41} \mathrm{H}_{78} \mathrm{NO}_{8} \mathrm{P}$ & Glycerophospholipids & $\begin{array}{l}\text { Plasma } \\
\text { membrane }\end{array}$ & 0.48 & 0.00 & 1.53 & 2.33 & & \\
\hline$\underline{P C[15: 1(9 Z) / 22: 4(7 Z, 10 Z, 13 Z, 16 Z)]}$ & LMGP01011460 & $\mathrm{C}_{45} \mathrm{H}_{80} \mathrm{NO}_{8} \mathrm{P}$ & Glycerophospholipids & $\begin{array}{l}\text { Plasma } \\
\text { membrane }\end{array}$ & 0.42 & 0.00 & 1.27 & 2.33 & & \\
\hline$\frac{\text { Butyl 4'-0-butanoyl-6-0- }}{\text { hexadecanoyl-neohesperidoside }}$ & LMSL05000005 & $\mathrm{C}_{36} \mathrm{H}_{66} \mathrm{O}_{12}$ & Saccharolipids & Surfactant & 0.22 & 0.00 & 1.24 & 2.33 & 0.14 & 0.00 \\
\hline$\underline{S M(d 16: 1 / 25: 0)}$ & LMSP03010076 & $\mathrm{C}_{46} \mathrm{H}_{93} \mathrm{~N}_{2} \mathrm{O}_{6} \mathrm{P}$ & Sphingolipids & $\begin{array}{l}\text { Absent in } \\
\text { animals }\end{array}$ & 0.67 & 0.00 & 1.53 & 2.33 & & \\
\hline
\end{tabular}

List of the mean fold-differences and respective $q$ values for the lipids being affected 2 weeks after tendon release (group T2) and L-carnitine administration (comparing groups T2 and T2-C) based on a $q$ value of $<5 \%$ (statistical analysis for microarrays). Lipid species in which levels are partially corrected in released muscle by L-carnitine administration are underlined. Note: Level alterations of glycerophospholipids were corrected in released muscle with L-carnitine administration.

identified 2 weeks after tendon release. Notably, the downregulation continued for two respiratory chain constituents up to 16 weeks after tendon release. These findings indicate that the capacity for mitochondrial respiration in infraspinatus muscle was reduced early (ie, 2 to 6 weeks), and possibly late (ie, 16 weeks), after tendon release. Reductions in ATP levels and increased inosinic acid levels, with muscle atrophy, like the ones observed in here (Supplemental Table S2), have been reported before to occur in rabbit muscle after tenotomy. ${ }^{44}$ Notably, the characterization of all expressed gene transcripts did not identify global alterations of gene ontologies associated with lipid biosynthesis, oxidation, and adipocyte differentiation in sheep infraspinatus muscle at 2 and 16 weeks after tendon release. Based on measures of selected adipogenesisassociated factors in rodent models for a massive rotator cuff tendon injury, it has been proposed that fatty atrophy is driven by the enhanced differentiation of adipocytes and/or degeneration of muscle-associated stem cells. ${ }^{6,45,46}$ Collectively, our observations indicate that a reduced capacity for the formation of high-energy reserves through the oxidative phosphorylation-mediated recharging of nucleosides (Supplemental Table S2) is a hallmark of the early pathologic process being induced by tendon release due to disuse before the accumulation of lipid becomes manifest.
The implication of a diminished capacity for mitochondrial respiration and metabolism in the accumulation of lipid in our sheep model is supported by the effects of L-carnitine on muscle metabolites and lipid species. For instance, with L-carnitine administration a trend for a reduced level of the $\beta$-oxidation-related $\mathrm{NAD}^{+47}$ was observed in muscle at 2 weeks after release when lipid species with longer carbon chain length and increased saturation levels accumulated (Supplemental Table S5). These alterations resemble those reported for fatty acid biosynthesis in bacterial ancestors of mitochondria in anaerobic conditions. ${ }^{48}$ Concomitantly, an opposite level regulation of 193 lipid species was observed between the released muscle and its contralateral control (Figure 7A). These differences included reduced levels of 99 fatty acyls in released muscle of L-carnitine-treated sheep when the corresponding levels were increased in contralateral muscle (Figure 7C). The former alterations are of interest, considering that the level of glycerol 3-phosphate that arises from the hydrolysis of triglycerides was increased in released muscle and contralateral muscle with carnitine administration (Supplemental Table S6). Because L-carnitine administration facilitates the import of fatty acid moieties into the mitochondrial matrix, ${ }^{49}$ the measured alterations in species of lipid metabolism were interpreted as to reflect the promotion of mitochondrial $\beta$-oxidation in the released muscle of $\mathrm{L}^{-}$ 
Table 3 A Sheep Model of Metabolites Being Affected by 2 Weeks of Tendon Release and L-Carnitine Administration

\begin{tabular}{|c|c|c|c|c|c|c|c|c|c|c|}
\hline \multirow[b]{2}{*}{ Description } & \multirow[b]{2}{*}{ Identifier } & \multirow[b]{2}{*}{ Formula } & \multirow[b]{2}{*}{$\begin{array}{l}\text { Substance } \\
\text { class }\end{array}$} & \multirow[b]{2}{*}{ Associated pathway } & \multicolumn{2}{|c|}{$\begin{array}{l}\text { Released versus } \\
\text { contralateral }\end{array}$} & \multicolumn{2}{|c|}{$\begin{array}{l}\text { L-Carnitine versus } \\
\text { no L-carnitine } \\
\text { (released) }\end{array}$} & \multicolumn{2}{|c|}{$\begin{array}{l}\text { L-Carnitine versus } \\
\text { no L-carnitine } \\
\text { (contralateral) }\end{array}$} \\
\hline & & & & & $\begin{array}{l}\text { Fold- } \\
\text { difference }\end{array}$ & $\begin{array}{l}q \\
\text { Value, } \\
\%\end{array}$ & $\begin{array}{l}\text { Fold- } \\
\text { difference }\end{array}$ & $\begin{array}{l}q \\
\text { Value, } \\
\%\end{array}$ & $\begin{array}{l}\text { Fold- } \\
\text { difference }\end{array}$ & $\begin{array}{l}q \\
\text { Value, } \\
\%\end{array}$ \\
\hline$\underline{\text { L-Glutamic acid }}$ & $\underline{\text { HMDB00148 }}$ & $\mathrm{C}_{5} \mathrm{H}_{9} \mathrm{NO}_{4}$ & Amino acid & $\begin{array}{l}\text { Alanine, aspartate and } \\
\text { glutamate metabolism }\end{array}$ & 2.00 & 1.43 & 0.67 & 0.00 & 0.36 & 0.00 \\
\hline Dimethylglycine & $\underline{\text { HMDB00092 }}$ & $\mathrm{C}_{4} \mathrm{H}_{9} \mathrm{NO}_{2}$ & Amino acid & $\begin{array}{l}\text { Glycine, serine and threonine } \\
\text { metabolism }\end{array}$ & 8.09 & 0.00 & 0.55 & 0.00 & 0.25 & 0.00 \\
\hline ATP & HMDB00538 & $\mathrm{C}_{10} \mathrm{H}_{16} \mathrm{~N}_{5} \mathrm{O}_{13} \mathrm{P}_{3}$ & Nucleoside & $\begin{array}{l}\text { High-energy reserve, oxidative } \\
\text { phosphorylation }\end{array}$ & 0.46 & 0.00 & 0.34 & 0.00 & 0.53 & 0.00 \\
\hline Adenosine $5^{\prime}$-diphosphate & HMDB01341 & $\mathrm{C}_{10} \mathrm{H}_{15} \mathrm{~N}_{5} \mathrm{O}_{10} \mathrm{P}_{2}$ & Nucleoside & $\begin{array}{l}\text { High-energy reserve, oxidative } \\
\text { phosphorylation }\end{array}$ & 0.59 & 0.00 & 0.42 & 0.00 & 0.47 & 0.00 \\
\hline Adenosine $2^{\prime}$-phosphate & HMDB11617 & $\mathrm{C}_{10} \mathrm{H}_{14} \mathrm{~N}_{5} \mathrm{O}_{7} \mathrm{P}$ & Nucleoside & $\begin{array}{l}\text { High-energy reserve, oxidative } \\
\text { phosphorylation }\end{array}$ & 0.53 & 3.62 & 0.40 & 0.00 & 0.52 & 0.00 \\
\hline Inosinic acid & HMDB00175 & $\mathrm{C}_{10} \mathrm{H}_{13} \mathrm{~N}_{4} \mathrm{O}_{8} \mathrm{P}$ & Nucleoside & $\begin{array}{l}\text { High-energy reserve, } \\
\text { ribonucleotide biosynthesis }\end{array}$ & 2.37 & 2.74 & 1.86 & 0.00 & 2.96 & 0.00 \\
\hline FAPy-adenine & HMDB04816 & $\mathrm{C}_{5} \mathrm{H}_{7} \mathrm{~N}_{5} \mathrm{O}$ & Nucleoside & 0xidized nucleotide & 0.74 & 3.79 & 0.60 & 0.00 & 0.62 & 0.00 \\
\hline Orotidine & HMDB00788 & $\mathrm{C}_{10} \mathrm{H}_{12} \mathrm{~N}_{2} \mathrm{O}_{8}$ & Nucleoside & Pyrimidine metabolism & 5.14 & 1.43 & 2.23 & 0.00 & 4.24 & 0.00 \\
\hline 2-Methylbutyrylglycine & HMDB00339 & $\mathrm{C}_{7} \mathrm{H}_{13} \mathrm{NO}_{3}$ & Lipid & Mitochondrial $\beta$-oxidation & 0.73 & 3.79 & 1.66 & 0.00 & 1.87 & 0.00 \\
\hline Irinotecan & HMDB14900 & $\mathrm{C}_{33} \mathrm{H}_{38} \mathrm{~N}_{4} \mathrm{O}_{6}$ & Medication & Drug metabolism & 1.48 & 3.42 & 0.52 & 0.00 & 0.12 & 0.00 \\
\hline$\overline{N \text {-desmethyl-mirtazapine }}$ & $\overline{\mathrm{HMDB} 60956}$ & $\mathrm{C}_{16} \mathrm{H}_{17} \mathrm{~N}_{3}$ & Medication & Drug metabolism & 1.66 & 3.42 & 0.75 & 4.55 & & \\
\hline$\frac{\text { 2-(4-Hydroxyphe-nyl) }}{\underline{\text { naphthalic anhydride }}}$ & $\overline{\mathrm{HMDB} 41121}$ & $\mathrm{C}_{18} \mathrm{H}_{10} \mathrm{O}_{4}$ & Food & Fruits & 3.21 & 3.42 & 0.60 & 4.55 & & \\
\hline Cloversaponin I & HMDB39892 & $\mathrm{C}_{36} \mathrm{H}_{56} \mathrm{O}_{9}$ & Food & Plant metabolism & 2.95 & 1.43 & 0.64 & 0.00 & 0.28 & 0.00 \\
\hline$\frac{\gamma-\mathrm{L}-\mathrm{Glutamyl}-\gamma \text {-L-glutamyl-L- }}{\underline{\text { methionine }}}$ & HMDB38674 & $\mathrm{C}_{15} \mathrm{H}_{25} \mathrm{~N}_{3} \mathrm{O}_{8} \mathrm{~S}$ & Food & Plant metabolism & 0.33 & 3.62 & 2.82 & 0.00 & 2.31 & 0.00 \\
\hline
\end{tabular}

List with the mean fold-difference and respective $q$ value for the metabolites being affected 2 weeks after tendon release (group T2) and $\mathrm{L}$-carnitine administration (comparing groups T2 and T2-C) based on a $q$ value of $<5 \%$ (statistical analysis for microarrays). Metabolites with levels that are partially corrected in released muscle by L-carnitine administration are underlined. Note: Level alterations of nucleosides (bold) being related to high-energy reserves through oxidative phosphorylation were exacerbated with L-carnitine administration.

carnitine-treated sheep. Collectively, these results specifically suggest that the overall (or total) capacity to couple the production of reduction equivalents (NADH) to ATP synthesis is reduced in infraspinatus muscle at 2 weeks after release due to a down-regulation of respiratory chain constituents. In this respect, it is noteworthy that the levels of the assessed proteins NDUFA9 and COX4I1, which reside in mitochondrial complexes I and IV, respectively, remained lowered at 16 weeks after tendon release (Figure 3). These observations thus emphasize that first, perturbations of mitochondria-related lipid metabolism can be detected at 2 weeks after tendon release in infraspinatus muscle, when the availability of nonoxidized substrates for mitochondrial metabolism, such as the amino acids, is elevated in consequence of the increased degradation of muscle protein. ${ }^{20}$

The observed effect of L-carnitine on the loss of infraspinatus muscle volume during the first 2 weeks after tendon release exceeded the threshold of one-half SD, which serves as an estimate for the minimally important difference, ${ }^{50}$ by 2.8 -fold. This difference indicates that the effect of oral L-carnitine administration may be clinically relevant in the prevention of early muscle atrophy.

The reversal of deteriorations in the level of the main free amino acid of skeletal muscle, glutamic acid, and plasma membrane-associated glycerophospholipids 2 weeks after tendon release by oral L-carnitine administration (Table 3) indicates that the counteraction of atrophy by L-carnitine administration involves a prevention of the degradation of muscle fiber protein and sarcolemmal constituents. ${ }^{51}$ These effects resemble the mitigation of reductions in the mean cross-sectional fiber area in rat slow-oxidative muscle during 2 weeks of muscle disuse by acetyl-L-carnitine supplementation. ${ }^{27}$ Conversely, to the suggestions by studies in rodents which demonstrated elevated mitochondrial transcript expression, ${ }^{27,28,31}$ the levels of only two of five measured respiratory chain components (ie, NDUFA9 and ATP5A1) tended to be higher with Lcarnitine administration in the sheep infraspinatus muscle (Figure 6). Together with the further lowered levels of oxidative phosphorylation-associated nucleosides in the released muscle (Table 3), our findings indicate that oral L-carnitine administration was not able to prevent the decline in the capacity for mitochondrial respiration. Intriguingly, lowered levels of oxidative phosphorylation-associated nucleosides and increased levels of inosinic acid were also observed in the contralateral muscle with L-carnitine administration, similar to what is observed with exhaustive exercise. ${ }^{47}$ Collectively, the pattern of effects indicates that the L-carnitine-associated prevention of infraspinatus muscle atrophy in sheep during the first 2 weeks after tendon release is mainly unrelated to effects on mitochondrial respiration. 
Intriguingly, the level of L-carnitine was sizably increased in the released infraspinatus muscle but lowered to the level in the contralateral muscle with the oral administration of L-carnitine (Supplemental Table S4). Increased levels of muscle carnitine have been reported to occur in the catabolic situation of fasting. ${ }^{52}$ These observations point out that the L-carnitine administration-mediated mitigation of muscle atrophy after tendon release is related to a normalization of the muscle levels of L-carnitine, rather than an increase in carnitine species. Concomitantly with the reduced levels of acyl-carnitines in the released muscle, including the major representant acetyl-L-carnitine, with L-carnitine administration, our observations indicate complex alterations in carnitine metabolism in atrophying muscle, which may also interact with limitations in muscle uptake of ingested carnitine. ${ }^{49}$

Carnitine supplementation at comparable or lower doses has been reported to suppress muscle atrophy during 2 weeks of disuse in rats. ${ }^{29}$ The underlying process has been discussed in terms of an improved nitrogen balance ${ }^{30}$ through a diminished myofibrillar protein degradation and a concomitantly increased drive for muscle protein synthesis. ${ }^{30,53,54}$ It has not been documented whether the former effects of carnitine supplementation comprise altered carnitine concentrations at the muscle level and/or systemic alterations in insulin signaling as shown in the liver, ${ }^{55}$ or a lowering of systemic inflammation. ${ }^{30}$ The prevented increase in glutamic acid levels in the released muscle by L-carnitine administration supports the view that L-carnitine halts muscle atrophy during the first 2 weeks by a reduction of muscle protein degradation via a mechanism that does not depend on a rise in the levels in L-carnitine and its acyl esters in the concerned muscle tissue.

A number of limitations may be considered to value our interpretations. First, our approach was that the conclusions on metabolites and lipid species being affected by tendon release were drawn from the comparison to its contralateral control, rather than in reference to samples collected prior to tendon release. This approach is explained by limits in the amount of tissue material that was available within the collected biopsy samples to conduct the extraction of metabolic compounds for ultrahigh-performance liquid chromatography with tandem mass spectrometry analysis on top of the analysis of RNA, proteins, and cell composition. Therefore, for the metabolomic/lipidomic measurements, tissue samples were used from the entire infraspinatus muscles, frozen immediately after extraction at the end of the experiment. Based on the previous characterization of the effects of 16 weeks of tendon release on lipid levels in the contralateral muscle, ${ }^{13}$ we are reasonably confident that an essential bias in the conclusions on the metabolic effects of tendon release can be excluded. This especially pertains to the effects of L-carnitine administration when both released and contralateral muscles were directly compared. Last, it may also be argued that the L-carnitine administration was pursued for a period that was too short, as lipid only began to accumulate beyond 6 weeks after tendon release (Figure 1C). The potential course of events was unknown when this study was designed, but nonetheless, evidence of aberrations in lipid metabolism was found in released muscle based on the probing of L-carnitine-inducible metabolic reactions at the 2-week time point when the protein levels for mitochondria respiratory chain constituents were down-regulated.

In summary, our results expose that atrophy of rotator cuff muscle subsequent to tendon release is reflected by the deterioration of slow oxidative muscle characteristics that take place before lipid accumulates in association with the loss of fast fiber area. The early down-regulation of the muscle level of respiratory chain proteins and high-energy phosphates 2 weeks after release recapitulates the alterations seen in locomotor muscle with reduced load-bearing muscle activity, ${ }^{23,25,56}$ corroborating with the aberrant accumulation of lipid species with L-carnitine administration the view that fatty degeneration of released rotator cuff is related to a reduced capacity for mitochondrial ATP synthesis. Oral administration of L-carnitine at the deployed high dose is identified as a potential nutraceutical countermeasure for preventing early atrophy of rotator cuff muscle by mitigating catabolism-related perturbations in glycerophospholipid and glutamic acid levels.

\section{Acknowledgments}

We thank Céline Ferrié, Marco Rohner, and Linus Oberholzer for assistance in the histologic analysis; and Philipp Kindt for animal care during housing of the animals.

\section{Author Contributions}

M.F., B.v.R., and C.G. designed the research; D.F., S.R., P.V., B.v.R., A.B., L.O., J.H., E.L., and K.W. performed the research; A.B.-N., L.O., J.H., and E.L. contributed analytic tools; M.F., D.F., S.R., L.O., J.H., and E.L. analyzed the data; M.F. wrote and revised the manuscript; and M.F., S.R., A.B.-N., L.O., and E.L. reviewed and revised the manuscript; M.F. is the guarantor of this work and, as such, had full access to all of the data in the study and takes responsibility for the integrity of the data and the accuracy of the data analysis.

\section{Supplemental Data}

Supplemental material for this article can be found at https://doi.org/10.1016/j.ajpath.2020.03.019.

\section{References}

1. Minagawa H, Yamamoto N, Abe H, Fukuda M, Seki N, Kikuchi K, Kijima H, Itoi E: Prevalence of symptomatic and asymptomatic rotator cuff tears in the general population: from mass-screening in one village. J Orthop 2013, 10:8-12 
2. Kolk A, Henseler JF, de Witte PB, van Zwet EW, van der Zwaal P, Visser CPJ, Nagels J, Nelissen R, de Groot JH: The effect of a rotator cuff tear and its size on three-dimensional shoulder motion. Clin Biomech (Bristol, Avon) 2017, 45:43-51

3. Lorbach O, Tompkins M: Rotator cuff: biology and current arthroscopic techniques. Knee Surg Sports Traumatol Arthrosc 2012, 20: 1003-1011

4. Sambandam SN, Khanna V, Gul A, Mounasamy V: Rotator cuff tears: an evidence based approach. World J Orthop 2015, 6:902-918

5. Shah SA, Kormpakis I, Cavinatto L, Killian ML, Thomopoulos S, Galatz LM: Rotator cuff muscle degeneration and tear severity related to myogenic, adipogenic, and atrophy genes in human muscle. J Orthop Res 2017, 35:2808-2814

6. Kim HM, Galatz LM, Lim C, Havlioglu N, Thomopoulos S: The effect of tear size and nerve injury on rotator cuff muscle fatty degeneration in a rodent animal model. J Shoulder Elbow Surg 2012, $21: 847-858$

7. Sciorati C, Clementi E, Manfredi AA, Rovere-Querini P: Fat deposition and accumulation in the damaged and inflamed skeletal muscle: cellular and molecular players. Cell Mol Life Sci 2015, 72: $2135-2156$

8. Fabbri M, Ciompi A, Lanzetti RM, Vadala A, Lupariello D, Iorio C, Serlorenzi P, Argento G, Ferretti A, De Carli A: Muscle atrophy and fatty infiltration in rotator cuff tears: can surgery stop muscular degenerative changes? J Orthop Sci 2016, 21:614-618

9. Bassett RW, Cofield RH: Acute tears of the rotator cuff. The timing of surgical repair. Clin Orthop Relat Res 1983:18-24

10. Deniz G, Kose O, Tugay A, Guler F, Turan A: Fatty degeneration and atrophy of the rotator cuff muscles after arthroscopic repair: does it improve, halt or deteriorate? Arch Orthop Trauma Surg 2014, 134: 985-990

11. Choo A, McCarthy M, Pichika R, Sato EJ, Lieber RL, Schenk S, Lane JG, Ward SR: Muscle gene expression patterns in human rotator cuff pathology. J Bone Joint Surg Am 2014, 96:1558-1565

12. Gibbons MC, Singh A, Engler AJ, Ward SR: The role of mechanobiology in progression of rotator cuff muscle atrophy and degeneration. J Orthop Res 2018, 36:546-556

13. Fluck M, Ruoss S, Mohl CB, Valdivieso P, Benn MC, von Rechenberg B, Laczko E, Hu J, Wieser K, Meyer DC, Gerber C: Genomic and lipidomic actions of nandrolone on detached rotator cuff muscle in sheep. J Steroid Biochem Mol Biol 2017, 165: 382-395

14. Itoigawa Y, Kishimoto KN, Sano H, Kaneko K, Itoi E: Molecular mechanism of fatty degeneration in rotator cuff muscle with tendon rupture. J Orthop Res 2011, 29:861-866

15. Schmutz S, Fuchs T, Regenfelder F, Steinmann P, Zumstein M, Fuchs B: Expression of atrophy mRNA relates to tendon tear size in supraspinatus muscle. Clin Orthop Relat Res 2009, 467:457-464

16. Laron D, Samagh SP, Liu X, Kim HT, Feeley BT: Muscle degeneration in rotator cuff tears. J Shoulder Elbow Surg 2012, 21:164-174

17. Hamrick MW, McGee-Lawrence ME, Frechette DM: Fatty infiltration of skeletal muscle: mechanisms and comparisons with bone marrow adiposity. Front Endocrinol (Lausanne) 2016, 7:69

18. Gerber C, Meyer DC, Fluck M, Benn MC, von Rechenberg B, Wieser K: Anabolic steroids reduce muscle degeneration associated with rotator cuff tendon release in sheep. Am J Sports Med 2015, 43: $2393-2400$

19. Vernon RG, Finley E, Taylor E: Fatty acid synthesis from amino acids in sheep adipose tissue. Comp Biochem Physiol B 1985, 82:133-136

20. Ji LL, Yeo D: Mitochondrial dysregulation and muscle disuse atrophy. F1000Res 2019, 8:F1000 Faculty Rev-1621

21. Powers SK, Wiggs MP, Duarte JA, Zergeroglu AM, Demirel HA: Mitochondrial signaling contributes to disuse muscle atrophy. Am J Physiol Endocrinol Metab 2012, 303:E31-E39

22. Hoeks J, Schrauwen P: Muscle mitochondria and insulin resistance: a human perspective. Trends Endocrinol Metab 2012, 23:444-450
23. Stevenson EJ, Giresi PG, Koncarevic A, Kandarian SC: Global analysis of gene expression patterns during disuse atrophy in rat skeletal muscle. J Physiol 2003, 551:33-48

24. Bey L, Akunuri N, Zhao P, Hoffman EP, Hamilton DG, Hamilton MT: Patterns of global gene expression in rat skeletal muscle during unloading and low-intensity ambulatory activity. Physiol Genomics 2003, 13:157-167

25. Loughna PT, Izumo S, Goldspink G, Nadal-Ginard B: Disuse and passive stretch cause rapid alterations in expression of developmental and adult contractile protein genes in skeletal muscle. Development 1990, 109:217-223

26. Yi-Wen Chen YW, Gregory CM, Scarborough MT, Shi R, Walter GA, Vandenborne K: Transcriptional pathways associated with skeletal muscle disuse atrophy in humans. Physiol Genomics 2007, 31:510-520

27. Cassano P, Fluck M, Giovanna Sciancalepore A, Pesce V, Calvani M, Hoppeler H, Cantatore P, Gadaleta MN: Muscle unloading potentiates the effects of acetyl-L carnitine on the slow oxidative muscle phenotype. Biofactors 2010, 36:70-77

28. Liu J, Peng Y, Feng Z, Shi W, Qu L, Li Y, Long J: Reloading functionally ameliorates disuse-induced muscle atrophy by reversing mitochondrial dysfunction, and similar benefits are gained by administering a combination of mitochondrial nutrients. Free Radic Biol Med 2014, 69:116-128

29. Jang J, Park J, Chang H, Lim K: L-carnitine supplement reduces skeletal muscle atrophy induced by prolonged hindlimb suspension in rats. Appl Physiol Nutr Metab 2016, 41:1240-1247

30. Ringseis R, Keller J, Eder K: Mechanisms underlying the antiwasting effect of L-carnitine supplementation under pathologic conditions: evidence from experimental and clinical studies. Eur J Nutr 2013, 52:1421-1442

31. Montesano A, Senesi P, Luzi L, Benedini S, Terruzzi I: Potential therapeutic role of L-carnitine in skeletal muscle oxidative stress and atrophy conditions. Oxid Med Cell Longev 2015, 2015:646171

32. Zhang X, Trevino MB, Wang M, Gardell SJ, Ayala JE, Han X, Kelly DP, Goodpaster BH, Vega RB, Coen PM: Impaired mitochondrial energetics characterize poor early recovery of muscle mass following hind limb unloading in old mice. J Gerontol A Biol Sci Med Sci 2018, 73:1313-1322

33. Neer CS 2nd, Craig EV, Fukuda H: Cuff-tear arthropathy. J Bone Joint Surg Am 1983, 65:1232-1244

34. Rowshan K, Hadley S, Pham K, Caiozzo V, Lee TQ, Gupta R: Development of fatty atrophy after neurologic and rotator cuff injuries in an animal model of rotator cuff pathology. J Bone Joint Surg Am 2010, 92:2270-2278

35. Liu X, Laron D, Natsuhara K, Manzano G, Kim HT, Feeley BT: A mouse model of massive rotator cuff tears. J Bone Joint Surg Am 2012, 94:e41

36. Gumucio JP, Qasawa AH, Ferrara PJ, Malik AN, Funai K, McDonagh B, Mendias CL: Reduced mitochondrial lipid oxidation leads to fat accumulation in myosteatosis. FASEB J 2019, 33: 7863-7881

37. Gerber C, Meyer DC, Schneeberger AG, Hoppeler H, von Rechenberg B: Effect of tendon release and delayed repair on the structure of the muscles of the rotator cuff: an experimental study in sheep. J Bone Joint Surg Am 2004, 86-A:1973-1982

38. Ruoss S, Kindt P, Oberholzer L, Rohner M, Jungck L, AbdelAziz S, Fitze D, Rosskopf A, Klein K, von Rechenberg B, Gerber C, Wieser K, Flück M: Inhibition of calpain delays early muscle atrophy after rotator cuff tendon release in sheep. Physiol Rep 2018, 6:e13833

39. Dobin A, Davis CA, Schlesinger F, Drenkow J, Zaleski C, Jha S, Batut P, Chaisson M, Gingeras TR: STAR: ultrafast universal RNAseq aligner. Bioinformatics 2013, 29:15-21

40. Schneider CA, Rasband WS, Eliceiri KW: NIH Image to ImageJ: 25 years of image analysis. Nat Methods 2012, 9:671-675 
41. Fluck M, Viecelli C, Bapst AM, Kasper S, Valdivieso P, Franchi MV, Ruoss S, Luthi JM, Buhler M, Claassen H, Hoppeler H, Gerber C: Knee extensors muscle plasticity over a 5 -years rehabilitation process after open knee surgery. Front Physiol 2018, 9:1343

42. Stein TP, Wade CE: Metabolic consequences of muscle disuse atrophy. J Nutr 2005, 135:1824S-1828S

43. Vamecq J, Dessein AF, Fontaine M, Briand G, Porchet N, Latruffe N, Andreolotti P, Cherkaoui-Malki M: Mitochondrial dysfunction and lipid homeostasis. Curr Drug Metab 2012, 13:1388-1400

44. Shchesno T: [Effect of tenotomy on the content of nucleic acids and phosphorus compounds in different types of skeletal muscles]. Vopr Med Khim 1966, 12:358-360

45. Liu X, Ning AY, Chang NC, Kim H, Nissenson R, Wang L, Feeley BT: Investigating the cellular origin of rotator cuff muscle fatty infiltration and fibrosis after injury. Muscles Ligaments Tendons J 2016, 6:6-15

46. Jensen AR, Kelley BV, Mosich GM, Ariniello A, Eliasberg CD, Vu B, Shah P, Devana SK, Murray IR, Peault B, Dar A, Petrigliano FA: Neer Award 2018: platelet-derived growth factor receptor alpha co-expression typifies a subset of platelet-derived growth factor receptor beta-positive progenitor cells that contribute to fatty degeneration and fibrosis of the murine rotator cuff. J Shoulder Elbow Surg 2018, 27:1149-1161

47. Norman B, Sollevi A, Kaijser L, Jansson E: ATP breakdown products in human skeletal muscle during prolonged exercise to exhaustion. Clin Physiol 1987, 7:503-510

48. Janssen HJ, Steinbuchel A: Fatty acid synthesis in Escherichia coli and its applications towards the production of fatty acid based biofuels. Biotechnol Biofuels 2014, 7:7
49. Stephens FB, Constantin-Teodosiu D, Greenhaff PL: New insights concerning the role of carnitine in the regulation of fuel metabolism in skeletal muscle. J Physiol 2007, 581:431-444

50. Norman GR, Sloan JA, Wyrwich KW: "Interpretation of changes in health-related quality of life: the remarkable universality of half a standard deviation". Med Care 2003, 41:582-592

51. Sreetama SC, Chandra G, Van der Meulen JH, Ahmad MM, Suzuki P, Bhuvanendran S, Nagaraju K, Hoffman EP, Jaiswal JK: Membrane stabilization by modified steroid offers a potential therapy for muscular dystrophy due to dysferlin deficit. Mol Ther 2018, 26: $2231-2242$

52. Rodriguez J, Bruyns J, Askanazi J, DiMauro W, Bordley J, Elwyn DH, Kinney JW: Carnitine metabolism during fasting in dogs. Surgery 1986, 99:684-687

53. Keller J, Couturier A, Haferkamp M, Most E, Eder K: Supplementation of carnitine leads to an activation of the IGF-1/PI3K/Akt signalling pathway and down regulates the E3 ligase MuRF1 in skeletal muscle of rats. Nutr Metab 2013, 10:28

54. Keller J, Ringseis R, Koc A, Lukas I, Kluge H, Eder K: Supplementation with L-carnitine downregulates genes of the ubiquitin proteasome system in the skeletal muscle and liver of piglets. Animal 2012, 6:70-78

55. Keller J, Ringseis R, Priebe S, Guthke R, Kluge H, Eder K: Effect of L-carnitine on the hepatic transcript profile in piglets as animal model. Nutr Metab (Lond) 2011, 8:76

56. Fluck M, Hoppeler H: Molecular basis of skeletal muscle plasticityfrom gene to form and function. Rev Physiol Biochem Pharmacol 2003, 146:159-216 